\title{
Non-separating planar chains in 4-connected graphs
}

\author{
Sean Curran $\quad$ Orlando Lee* Xingxing $\mathrm{Yu}^{\dagger}$
}

June 27, 2002

School of Mathematics

Georgia Institute of Technology

Atlanta, Georgia 30332

\begin{abstract}
In this paper, we describe an $O\left(|V(G)|^{2}\right)$ algorithm for finding a "non-separating planar chain" in a 4-connected graph $G$, which will be used to decompose an arbitrary 4-connected graph into "planar chains". This work was motivated by the study of a multi-tree approach to reliability in distributed networks, as well as the study of non-separating induced paths in highly connected graphs.
\end{abstract}

*Supported by CNPq (Proc: 200611/00-3).

${ }^{\dagger}$ Partially supported by NSF grant DMS 9970527 


\section{Introduction}

Let $G=(V(G), E(G))$ denote a graph with vertex set $V(G)$ and edge set $E(G)$. We use the notation $x y$ (or $y x$ ) to represent an edge with ends $x$ and $y$. For any $S \subseteq V(G)$, let $G[S]$ denote the subgraph of $G$ with $V(G[S])=S$ and $E(G[S])$ consisting of the edges of $G$ with both ends in $S$; we say that $G[S]$ is the subgraph of $G$ induced by $S$. Let $G-S$ denote $G[V(G)-S]$. A subgraph $H$ of $G$ is an induced subgraph of $G$ if $G[V(H)]=H$. We also say that $H$ is induced in $G$. A graph $G$ is $k$-connected, where $k$ is a positive integer, if $|V(G)| \geq k+1$ at least $k+1$ and, for any $S \subset V(G)$ with $|S| \leq k-1, G-S$ is connected. A subgraph $H$ of $G$ is non-separating if $G-V(H)$ is connected.

In 1984, Itai and Rodeh [9] proposed a multi-tree approach to reliability in distributed networks. Let $G$ be a graph and $r \in V(G)$. We may view $G$ as a distributed network with a root $r$, and the vertices of $G$ as processors. A fault-tolerant communication scheme can be designed for this network if we are able to find spanning trees of $G$ which are "independent" $[5,9]$. For a tree $T$ and $x, y \in V(T)$, let $T[x, y]$ denote the unique path from $x$ to $y$ in $T$. A rooted tree $T$ is a tree with a specified vertex called the root of $T$. Let $T$ and $T^{\prime}$ be trees in a graph rooted at $r$. We say that $T$ and $T^{\prime}$ are independent if for each vertex $x \in V(T) \cap V\left(T^{\prime}\right)$, the paths $T[r, x]$ and $T^{\prime}[r, x]$ have no vertex in common except for $r$ and $x$.

Itai and Rodeh [9] developed a linear time algorithm that given any vertex $r$ in a 2-connected graph $G$, finds two independent spanning trees of $G$ rooted at $r$. Later, Cheryian and Maheshwari [2] proved that for any vertex $r$ in a 3-connected graph $G$, there exist three independent spanning trees of $G$ rooted at $r$. Furthermore, they gave an $O\left(|V(G)|^{2}\right)$ algorithm for finding these trees. Itai and Zehavi [10] proved independently that every 3-connected graph contains three independent spanning trees (rooted at any vertex), and they conjectured the following.

(1.1) Conjecture. Let $G$ be a $k$-connected graph and let $r \in V(G)$. Then there exist $k$ independent spanning trees of $G$ rooted at $r$.

A contractible edge in a $k$-connected graph is an edge whose contraction results in a new $k$-connected graph. Itai and Zehavi's proof for the 3 -connected case relies on the existence of a contractible edge. On the other hand, for every $k \geq 4$ there exist infinitely many $k$-connected graphs with no contractible edges. In view of this fact, it would be interesting to know if (1.1) holds for $k=4$. The 4-connected case of (1.1) is also important in terms of applications, since four independent spanning trees ensure at a reasonable cost a higher degree of reliability in distributed networks. Huck [7] proved (1.1) for planar 4-connected graphs. Miura, Nakano, Nishizeki and Takahashi [13] gave a linear algorithm for finding four independent rooted spanning trees in a planar 4-connected graph. 
Itai and Rodeh's algorithm [9] for constructing two independent spanning trees relies on "ear decompositions" of graphs. Cheriyan and Maheshwari [2] used the concept of "non-separating ear decomposition" to construct three independent spanning trees in 3 -connected graphs. The first step in their approach is to find a non-separating cycle which "avoids" a given vertex. A cycle $C$ avoids a vertex $v$ if $v \notin V(C)$.

(1.2) Theorem. Let $G$ be a 3-connected graph, let $e \in E(G)$, and let $u \in V(G)$ be nonincident to $e$. Then $G$ has a non-separating cycle through $e$ and avoiding $u$. Moreover, such a cycle can be found in $O(|V(G)|+|E(G)|)$ time.

The existence of a non-separating cycle in (1.2) was proved by Tutte [20], and the algorithmic part was done by Cheryian and Maheshwari ([2], Theorem 5). In general, it is not true that given an edge $e$ in a 4-connected graph $G$, there exists an induced cycle $C$ through $e$ such that $G-V(C)$ is 2-connected. However, we will see that this is possible when $C$ is a "chain". (It seems to me that this is not exactly true, because we cannot remove the vertex $r$ )

In this paper we are concerned with the problem of finding a "non-separating planar chain" in a 4-connected graph. A "non-separating planar chain" can be viewed as a generalization of the concept of a non-separating path. We give an efficient algorithm for solving this problem. Our result has some interesting consequences (Section 4) and will be used in a forthcoming paper to decompose an arbitrary 4-connected graph into "planar chains". In order to describe precusely our result, we need to introduce the concept of "chain" and "planar chain".

A block of a graph $G$ is either a maximal 2-connected subgraph of $G$, or a subgraph of $G$ induced by a cut edge. A block is nontrivial if it is 2-connected, and trivial otherwise.

(1.3) Definition. A connected graph $H$ is a chain if its blocks can be labeled as $B_{1}, \ldots, B_{k}$, where $k \geq 1$ is an integer, and its cut vertices can be labeled as $v_{1}, \ldots, v_{k-1}$ such that

(i) $V\left(B_{i}\right) \cap V\left(B_{i+1}\right)=\left\{v_{i}\right\}$ for $1 \leq i \leq k-1$, and

(ii) $V\left(B_{i}\right) \cap V\left(B_{j}\right)=\emptyset$ if $|i-j| \geq 2$ and $1 \leq i, j \leq k$.

We let $H:=B_{1} v_{1} B_{2} v_{2} \ldots v_{k-1} B_{k}$ denote this situation. If $k \geq 2, v_{0} \in V\left(B_{1}\right)-\left\{v_{1}\right\}$ and $v_{k} \in V\left(B_{k}\right)-\left\{v_{k-1}\right\}$, then we say that $H$ connects $v_{0}$ and $v_{k}$. If $k=1, v_{0}, v_{k} \in V\left(B_{1}\right)$ and $v_{0} \neq v_{k}$, then we also say that $H$ connects $v_{0}$ and $v_{k}$. In both cases, we say that $H$ is a $v_{0}-v_{k}$ chain, and we denote this by $H:=v_{0} B_{1} v_{1} \ldots v_{k-1} B_{k} v_{k}$. We usually fix $v_{0}$ and $v_{k}$, and we refer to them as the ends of $H_{i}$. See Figure 1 for an example.

A plane graph is a graph which is drawn in the plane with no pair of edges crossing. Let $G$ be a graph with distinct vertices $a, b, c$ and $d$. We say that the quintuple 


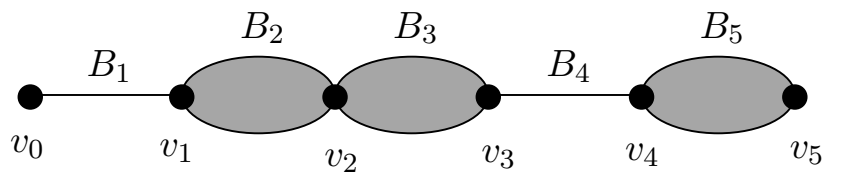

Figure 1: Example of a chain.

$(G, a, b, c, d)$ is planar if $G$ can be drawn in a closed disc in the plane with no pair of edges crossing such that $a, b, c, d$ occur on the boundary of the disc in this cyclic order.

For a graph $G$ and $x, y \in V(G)$ let $G-x y$ denote the graph with vertex set $V(G)$ and edge set $E(G)-\{x y\}$ (note that $x y$ may not be an edge of $G$ ).

(1.4) Definition. Let $G$ be a graph and let $H:=v_{0} B_{1} v_{1} \ldots v_{k-1} B_{k} v_{k}$ be a chain. If $H$ is an induced subgraph of $G$, then we say that $H$ is a chain in $G$. We say that $H$ is a planar chain in $G$ if, for each $1 \leq i \leq k$ with $\left|V\left(B_{i}\right)\right| \geq 3$ (or equivalently, $B_{i}$ is 2-connected), there exist distinct vertices $x_{i}, y_{i} \in V(G)-V(H)$ such that $\left(G\left[V\left(B_{i}\right) \cup\left\{x_{i}, y_{i}\right\}\right]-x_{i} y_{i}, x_{i}, v_{i-1}, y_{i}, v_{i}\right)$ is planar, and $B_{i}-\left\{v_{i-1}, v_{i}\right\}$ is a component of $G-\left\{x_{i}, y_{i}, v_{i-1}, v_{i}\right\}$. We also say that $H$ is a planar $v_{0}-v_{k}$ chain. See Figure 2 for an illustration.

(1.5) Definition. Let $G$ be a graph, $S \subseteq V(G)$ and $k$ be a positive integer. We say that $G$ is $(k, S)$-connected if $|V(G)| \geq|S|+1, G$ is connected, and for any $T \subset V(G)$ with $|T| \leq k-1$, every component of $G-T$ contains an element of $S$.

This definition is partially motivated by the following observation. Let $G$ be a $k$ connected graph, let $S \subseteq V(G)$ and let $K$ be a component of $G-S$. Then $G[V(K) \cup S]$ is $(k, S)$-connected.

Now we are ready to describe the main result of this paper. It is stated in a form which can be conveniently used in a forthcoming paper. See Figure 5 for an illustration of the hypothesis of the theorem.

(1.6) Theorem. Let $G$ be a graph, let $a, b$ be distinct vertices of $G$, let $P$ be a nonseparating induced $a-b$ path in $G$ avoiding $r$, let $B_{P}$ be a nontrivial block of $G-V(P)$, and let $X_{P}$ be the set consisting of the cut vertices of $G-V(P)$ contained in $V\left(B_{P}\right)$ and the neighbors of $V(P)$ contained in $V\left(B_{P}\right)$. Suppose $G-\left(V\left(B_{P}\right)-X_{P}\right)$ is $\left(4, X_{P} \cup\{a, b\}\right)$ connected. Then there exists a planar $a-b$ chain $H$ in $G$ such that $B_{P} \subseteq G-V(H)$ and $G-V(H)$ is 2-connected. Moreover, such a chain can be found in in $O(|V(G)||E(G)|)$ time. 

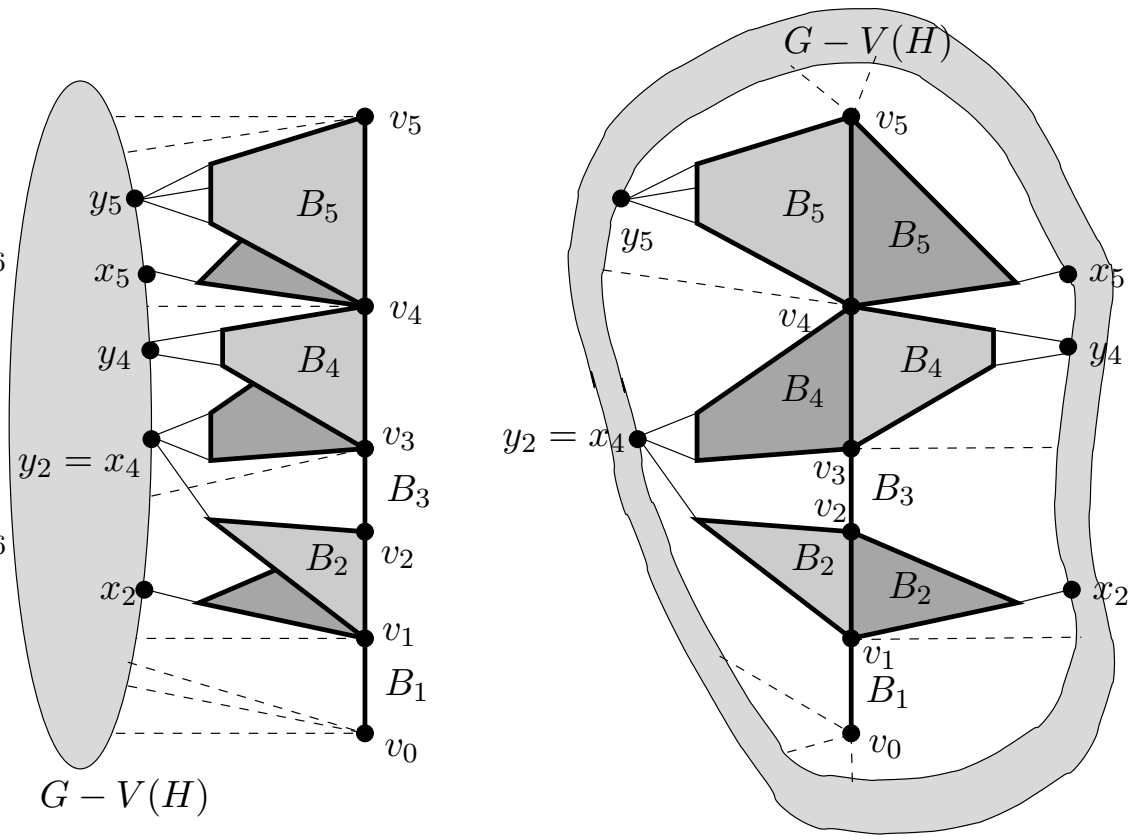

Figure 2: Two drawings of a planar chain $H:=v_{0} B_{1} v_{1} B_{2} v_{2} B_{3} v_{3} B_{4} v_{4} B_{5} v_{5}$ in a graph $G$. The dashed edges may exist or not, but they are not part of $H$.

An interesting consequence of (1.6) (Corollary (4.9)) says that if $G$ is a 4-connected graph and $r \in V(G)$, then there exists a cycle $C$ in $G$ through $r$ such that $G-(V(C)-\{r\})$ is 2-connected. This is related to an open problem posed by Lovász [12]. See Section 4.

The rest of this paper is organized as follows. In the remainder of this section we establish the notation we use throughout the paper. In Section 2 we give several auxiliary lemmas. These lemmas concern the existence of certain non-separating paths in graphs with some connectivity constraints. In Section 3 we prove (1.6). In Section 4 we prove several consequences of (1.6).

We use $A:=B$ to rename $B$ as $A$, or to define $A$ as $B$.

Let $G$ be a graph. For $S \subseteq V(G)$, let $N_{G}(S):=\{x \in V(G)-S: x y \in$ $E(G)$, for some $y \in S\}$. For a subgraph $H$ of $G$, we write $N_{G}(H):=N_{G}(V(H))$. When $S=\{x\}$, we let $N_{G}(x):=N_{G}(\{x\})$. When there exists no ambiguity, we may simply use $N(S), N(H)$ and $N(x)$, instead of $N_{G}(S), N_{G}(H)$ and $N_{G}(x)$, respectively. For a set $F$ of 2-element subsets of $V(G)$, let $G+F$ denote the graph with vertex set $V(G)$ and edge set $E(G) \cup F$. If $F:=\{x y\}$, let $G+x y:=G+F$.

We describe a path in $G$ as a sequence $P=\left(v_{1}, v_{2}, \ldots, v_{k}\right)$ of distinct vertices of $G$ such that $v_{i} v_{i+1} \in E(G), 1 \leq i \leq k-1$. The vertices $v_{1}$ and $v_{k}$ are called the 
ends of the path $P$. The vertices in $V(P)-\left\{v_{1}, v_{k}\right\}$ are called the internal vertices of $P$. For $1 \leq i \leq j \leq k$, let $P\left[v_{i}, v_{j}\right]:=\left(v_{i}, \ldots, v_{j}\right)$, and for $1 \leq i<j \leq k$, let $P\left(v_{i}, v_{j}\right):=P\left[v_{i+1}, v_{j-1}\right]$. For $A, B \subseteq V(G)$, we say that a path $P$ is an $A$ - $B$ path if one end of $P$ is in $A$, the other end is in $B$, and no internal vertex of $P$ is in $A \cup B$. If $P$ is a path with ends $a$ and $b$, we say that $P$ is a path from $a$ to $b$, or $P$ is an $a-b$ path. Two paths $P$ and $Q$ are disjoint if $V(P) \cap V(Q)=\emptyset$. Two paths are internally disjoint if no internal vertex of one is contained in the other. Given a path $P$ in $G$ and a set $S \subset V(G)$ (respectively, a subgraph $S$ of $G$ ), we say that $P$ is internally disjoint from $S$ if no internal vertex of $P$ is contained in $S$ (respectively, $V(S)$ ). We also describe a cycle in $G$ as a sequence $C=\left(v_{1}, v_{2}, \ldots, v_{k}, v_{1}\right)$ such that the vertices $v_{1}, \ldots, v_{k}$ are distinct, $v_{i} v_{i+1} \in E(G)$, for $1 \leq i \leq k-1$, and $v_{k} v_{1} \in E(G)$.

\section{Non-Separating paths}

In trying to find a non-separating planar chain, we need to be able to find efficiently disjoint paths and non-separating paths in graphs which satisfy certain connectivity conditions. The purpose of this section is to provide auxiliary lemmas (and algorithms) to deal with these problems.

The two disjoint paths problem can be defined as follows: given a graph $G$ and distinct vertices $a, b, c, d$ of $G$, find disjoint paths from $a$ to $b$, and from $c$ to $d$, respectively, or certify that they do not exist.

Seymour [15], Thomassen [18] and Shiloach [16] solved independently the two disjoint paths problem. We state Seymour's version ([15], Theorem 4.1).

(2.1) Theorem. Let $a, b, c, d$ be distinct vertices of a graph $G$. Then exactly one of the following holds:

(1) G contains disjoint paths from a to $b$, and from $c$ to $d$, respectively, or

(2) for some integer $k \geq 0$, there exist pairwise disjoint sets $A_{1}, \ldots, A_{k} \subseteq V(G)-$ $\{a, b, c, d\}$ such that

- for $1 \leq i \neq j \leq k, N_{G}\left(A_{i}\right) \cap A_{j}=\emptyset$,

- for $1 \leq i \leq k,\left|N_{G}\left(A_{i}\right)\right| \leq 3$, and

- if $G^{\prime}$ is the graph obtained from $G$ by, for each $i$, deleting $A_{i}$ and adding new edges joining every pair of distinct vertices in $N_{G}\left(A_{i}\right)$, and also, adding the edges $a b$ and $c d$, then $G^{\prime}$ can be drawn in the plane with no pairs of edges crossing except $a b$ and $c d$, which cross once.

Let $G$ be a graph and $S:=\{a, b, c, d\} \subseteq V(G)$. Shiloach $[16]$ gave an $O(|V(G) \| E(G)|)$ algorithm for the two disjoint paths problem. We need to solve a special case of the two 
disjoint paths problem, namely, when $G$ is $(4, S)$-connected. We show in the appendix that Shiloach's algorithm can solve the two disjoint paths problem in $O(|V(G)|+|E(G)|)$ time for $(4, S)$-connected graphs.

(2.2) Lemma. Let $G$ be a graph and let $S:=\{a, b, c, d\} \subset V(G)$. Suppose that $G$ is $(4, S)$-connected. Then exactly one of the following holds:

(1) there exist disjoint paths from $a$ to $b$ and from $c$ to $d$, respectively, or

(2) $(G, a, c, b, d)$ is planar.

Moreover, one can in $O(|V(G)|+|E(G)|)$ time find paths as in (1) or certify that (2) holds.

Next, we prove Lemmas (2.3) and (2.4) concerning non-separating induced paths in graphs with certain connectivity properties. We also show how to find these paths efficiently. These lemmas will be used extensively.

(2.3) Lemma. Let $G$ be a connected graph, $S \subseteq V(G),\left\{a, a^{\prime}\right\} \subseteq S$, and let $P$ be an a-a' path in G. Suppose

(i) $G$ is $(3, S)$-connected, and

(ii) $S-\left\{a, a^{\prime}\right\}$ is contained in a component $U$ of $G-V(P)$.

Then there exists a non-separating induced $a-a^{\prime}$ path $P^{\prime}$ in $G$ such that $V\left(P^{\prime}\right) \cap V(U)=\emptyset$. Moreover, such a path can be found in $O(|V(G)|+|E(G)|)$ time.

Proof. We may assume that $P$ is induced, otherwise, we can find in $O(|V(G)|+|E(G)|)$ time an induced $a-a^{\prime}$ path satisfying (ii). If $P$ is non-separating, then $P^{\prime}:=P$ is the required path. If $|V(P)|=2$ then by (i) every component of $G-V(P)$ contains a vertex of $S$, and so by (ii) $G-V(P)=U$, which implies that $P$ is non-separating. So we may assume that $|V(P)| \geq 3$ and $G-V(P)$ is not connected.

Let $G^{\prime}$ be the graph obtained from $G$ by contracting $U$ to a single vertex $u$, by adding the edges $a a^{\prime}, u a$ and $u a^{\prime}$, and by removing multiple edges. See Figure 3. Note that $a, a^{\prime}$ belong to the cycle $P+a a^{\prime}$. We claim that $H:=G^{\prime}-u$ is 2-connected. Suppose for a contradiction that there exists $v \in V(H)$ such that $H-v$ is disconnected. Since $a, a^{\prime}$ are vertices of $P+a a^{\prime}$ which is a cycle in $H$, there exists a component $K$ in $H-v$ which does not contain any vertex of $P$. But then $K$ is a component of $G-v$ which does not contain any vertex in $S$. This contradicts (i). Thus, $G^{\prime}-u$ is 2-connected.

In fact, $G^{\prime}$ must be 3 -connected. Suppose for a contradiction that $G^{\prime}$ is not 3connected. Then there is a vertex cut $T$ in $G^{\prime}$ with $|T| \leq 2$. Since $G^{\prime}-u$ is 2 -connected, $u \notin T$. Moreover, since $\left\{u, a, a^{\prime}\right\}$ induces a triangle, there exists a component $K$ of $G^{\prime}-T$ 


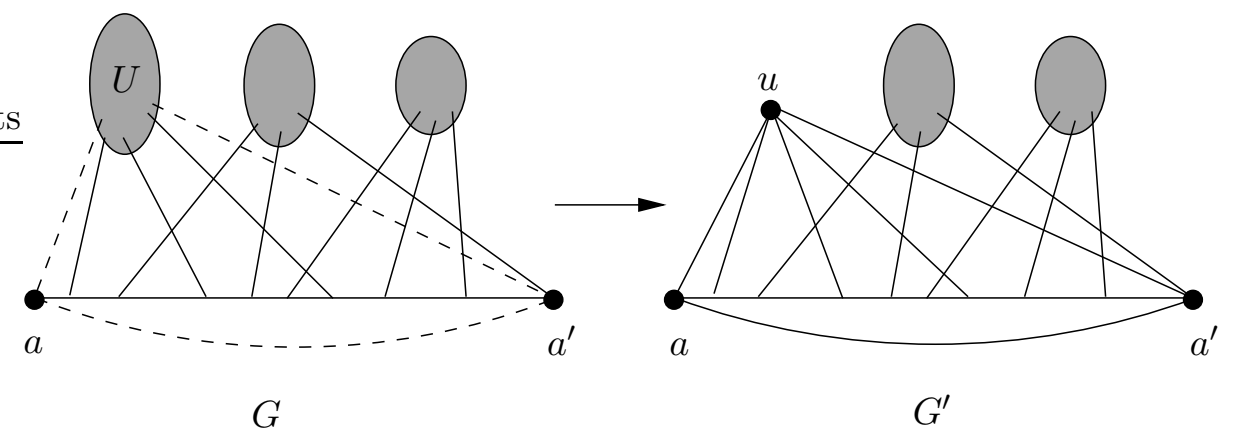

Figure 3: Graphs $G$ and $G^{\prime}$ in the proof of (2.3).

which does not contain any of $u, a, a^{\prime}$. But then $K$ is also a component of $G-T$ which does not contain any element of $S$, which contradicts (i). Hence, $G^{\prime}$ is 3-connected.

By Theorem (1.2) (with $G^{\prime}, a a^{\prime}, u$ as $G, e, u$ respectively), there exists a non-separating induced cycle $C$ in $G^{\prime}$ containing $a a^{\prime}$ and avoiding $u$. Moreover, such a cycle can be found in $O\left(\left|V\left(G^{\prime}\right)\right|+\left|E\left(G^{\prime}\right)\right|\right)$ time (and hence, in $O(|V(G)|+|E(G)|)$ time). Thus, $P^{\prime}:=C-a a^{\prime}$ is a non-separating induced path in $G$ such that $V\left(P^{\prime}\right) \cap V(U)=\emptyset$.

The next result is a strengthening of Lemma (2.2).

(2.4) Lemma. Let $G$ be a graph and $S:=\left\{a, a^{\prime}, b, b^{\prime}\right\} \subseteq V(G)$. Suppose that $G$ is $(4, S)$-connected. Then exactly one of the following holds:

(1) there exists a non-separating induced $a-a^{\prime}$ path $P^{\prime}$ in $G$ such that $V\left(P^{\prime}\right) \cap\left\{b, b^{\prime}\right\}=\emptyset$, or

(2) $\left(G, a, b, a^{\prime}, b^{\prime}\right)$ is planar.

Moreover, one can in $O(|V(G)|+|E(G)|)$ time find a path as in (1) or certify that (2) holds.

Proof. By Lemma (2.2), either (a) there exist disjoint paths $P$ and $Q$ in $G$ from $a$ to $a^{\prime}$ and from $b$ to $b^{\prime}$, respectively, or (b) $\left(G, a, b, a^{\prime}, b^{\prime}\right)$ is planar. Moreover, one can in $O(|V(G)|+|E(G)|)$ time find paths as in (a) or certify that (b) holds. If (b) holds, then (2) holds. So assume (a) holds. Let $U$ be the component of $G-V(P)$ containing $S-\left\{a, a^{\prime}\right\}=$ $\left\{b, b^{\prime}\right\}$. Since $G$ is $(4, S)$-connected (and hence, $(3, S)$-connected), $G, P, S, U$ and $\left\{a, a^{\prime}\right\}$ satisfy the hypothesis of (2.3). Thus, by (2.3) there exists a non-separating $a-a^{\prime}$ path $P^{\prime}$ such that $V\left(P^{\prime}\right) \cap V(U)=\emptyset$, and such a path can be found in $O(|V(G)|+|E(G)|)$ time. Hence, $V\left(P^{\prime}\right) \cap\left\{b, b^{\prime}\right\}=\emptyset$, and $P^{\prime}$ satisfies (1) of (2.4). 
To prove the final lemma of this section, we need the following result of Cheriyan and Maheshwari ([2], p. 516), which is in fact, the core of the linear algorithm in [2] for finding a non-separating induced cycle as described in Theorem (1.2).

(2.5) Theorem. Let $G$ be a 3-connected graph, let $a a^{\prime} \in E(G)$, and let $C$ be a nonseparating induced cycle in $G$ containing $a a^{\prime}$. Then there exists another non-separating induced cycle $C^{\prime}$ in $G$ such that $V\left(C^{\prime}\right) \cap V(C)=\left\{a, a^{\prime}\right\}$ and $E\left(C^{\prime}\right) \cap E(C)=\left\{a a^{\prime}\right\}$. Moreover, such a cycle can be found in $O(|V(G)|+|E(G)|)$ time.

The next result is in the same spirit as of (2.5), but we relax the 3-connectivity condition. This result is more convenient to use.

(2.6) Lemma. Let $G$ be a connected graph, let $a, a^{\prime}$ be distinct vertices of $G$ with degree at least two, and let $P$ be a non-separating induced $a-a^{\prime}$ path in $G$. Suppose that $G$ is $(3, V(P))$-connected. Then there exists another non-separating induced $a-a^{\prime}$ path $P^{\prime}$ in $G$ such that $V\left(P^{\prime}\right) \cap V(P)=\left\{a, a^{\prime}\right\}$ and $E\left(P^{\prime}\right) \cap E(P)=\emptyset$. Moreover, such a path can be found in $O(|V(G)|+|E(G)|)$ time.

Proof. For convenience, let $H:=G-V(P)$. Since $P$ is a non-separating path in $G, H$ is connected. Morever, $a$ and $a^{\prime}$ have a neighbor in $V(H)$ because both have degree at least two in $G$ and $P$ is induced. Let $v_{1}=a, v_{2}, \ldots, v_{k}=a^{\prime}$ be the neighbors of $H$ on $P$ in this order from $a$ to $a^{\prime}$ (see Figure 4 for an illustration). Note that $k \geq 3$ because $G$ is $(3, V(P))$-connected. Let $G^{\prime}$ be the graph obtained from $G$ by adding the edge $a a^{\prime}$, and

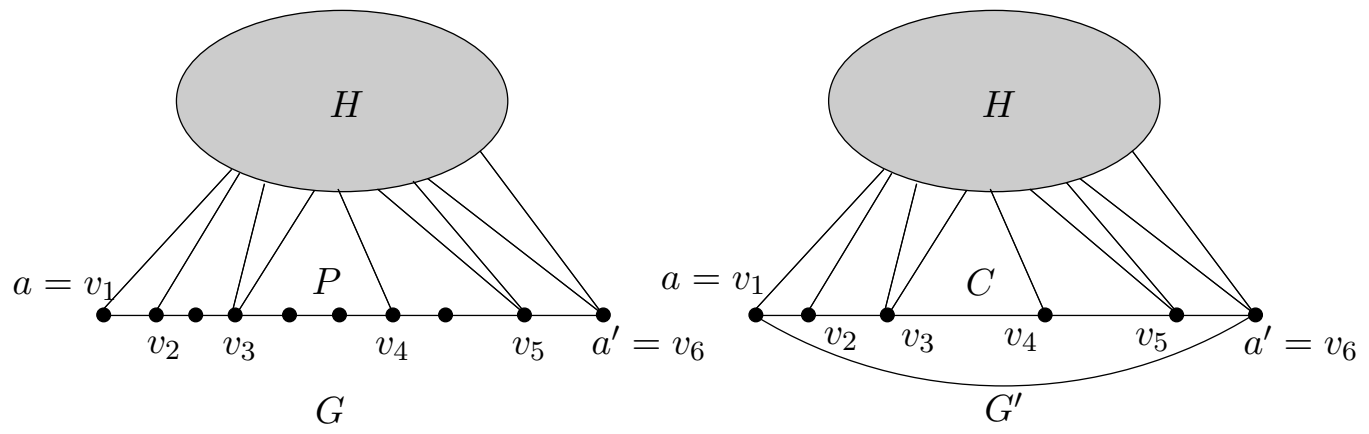

Figure 4: $G$ and $G^{\prime}$ as in the proof of Lemma (2.6).

by replacing, for each $1 \leq i \leq k-1$, the path $P\left[v_{i}, v_{i+1}\right]$ by an edge $v_{i} v_{i+1}$. Note that $C:=G^{\prime}-V(H)$ is a cycle in $G^{\prime}$.

We claim that $G^{\prime}$ is 3-connected. Suppose for a contradiction that $G^{\prime}$ is not 3connected. Then there is a vertex cut $T$ in $G^{\prime}$ with $|T| \leq 2$. Note that $T \nsubseteq V(C)$, 
since $P$ is non-separating and every vertex of $C$ has a neighbor in $H$. But then $G^{\prime}-T$ has a component $K$ such that $V(K) \cap V(C)=V(K) \cap V(P)=\emptyset$. Hence, $K$ is also a component of $G-T$ with $V(K) \cap V(P)=\emptyset$, which contradicts the assumption that $G$ is $(3, V(P))$-connected. Hence, $G^{\prime}$ is 3 -connected.

By (2.5) (with $G^{\prime}, C, a, a^{\prime}$ as $G, C, a, a^{\prime}$ respectively), there exists a non-separating cycle $C^{\prime}$ in $G^{\prime}$ such that $V\left(C^{\prime}\right) \cap V(C)=\left\{a, a^{\prime}\right\}$ and $E\left(C^{\prime}\right) \cap E(C)=\left\{a a^{\prime}\right\}$. Moreover, such a cycle can be found in $O\left(\left|V\left(G^{\prime}\right)\right|+\left|E\left(G^{\prime}\right)\right|\right)$ time (and hence, in $O(|V(G)|+|E(G)|$ ) time). Thus, $P^{\prime}:=C^{\prime}-a a^{\prime}$ is a non-separating induced $a-a^{\prime}$ path in $G$ such that $V\left(P^{\prime}\right) \cap V(P)=\left\{a, a^{\prime}\right\}$ and $E\left(P^{\prime}\right) \cap E(P)=\emptyset$.

\section{$3 \quad$ Non-separating chains}

Our goal is to design an algorithm that solves the following problem: given $G, a, b, P, B_{P}$ as in (1.6), find a planar $a-b$ chain $H$ in $G$ such that $G-V(H)$ is 2connected and $V\left(B_{P}\right) \subseteq V(G)-V(H)$. For convenience, we fix the following notation throughout this section.

(3.1) Notation and assumptions. Let $G$ be a graph, let $r, a, b$ be distinct vertices of $G$, let $P$ be a non-separating induced $a-b$ path in $G$, let $B_{P}$ be a nontrivial block of $G-V(P)$, and let $X_{P}$ be the set consisting of cut vertices of $G-V(P)$ contained in $V\left(B_{P}\right)$ and the neighbors of $V(P)$ contained in $V\left(B_{P}\right)$. Suppose that $G-\left(V\left(B_{P}\right)-X_{P}\right)$ is $\left(4, X_{P} \cup\{a, b\}\right)$-connected. See Figure 5 .

Let $\mathcal{P}_{P}$ be the set of non-separating induced $a-b$ paths $P^{\prime}$ in $G$ such that $B_{P} \subseteq$ $G-V\left(P^{\prime}\right)$. Note that $P \in \mathcal{P}_{P}$. For each $P^{\prime} \in \mathcal{P}_{P}$ let $B_{P^{\prime}}$ denote the nontrivial block of $G-V\left(P^{\prime}\right)$ which contains $B_{P}$. We say that $P^{\prime} \in \mathcal{P}_{P}$ is a $B_{P}$-augmenting path if $\left|V\left(B_{P}\right)\right|<\left|V\left(B_{P^{\prime}}\right)\right|$.

The next lemma shows that the paths in $\mathcal{P}_{P}$ are well-behaved.

(3.2) Lemma. Let $P^{\prime} \in \mathcal{P}_{P}$. Let $X_{P^{\prime}}$ be the set consisting of the cut vertices of $G-V\left(P^{\prime}\right)$ contained in $B_{P^{\prime}}$ and the neighbors of $V\left(P^{\prime}\right)$ in $B_{P^{\prime}}$. Then $G-\left(V\left(B_{P^{\prime}}\right)-X_{P^{\prime}}\right)$ is $\left(4, X_{P^{\prime}} \cup\{a, b\}\right)$-connected.

Proof. For convenience, let $G^{\prime}:=G-\left(V\left(B_{P^{\prime}}\right)-X_{P^{\prime}}\right)$. Suppose there exists $T \subset V\left(G^{\prime}\right)$ with $|T| \leq 3$ and there exists some component $K$ of $G^{\prime}-T$ such that $V(K) \cap\left(X_{P^{\prime}} \cup\right.$ $\{a, b\})=\emptyset$. Since $V\left(B_{P}\right) \subseteq V\left(B_{P^{\prime}}\right)$, for every $x \in X_{P}$, either $x \in X_{P^{\prime}}$ or $x \notin V\left(G^{\prime}\right)$. Thus, $V(K) \cap X_{P}=\emptyset$. But then, $K$ is a component of $\left(G-\left(V\left(B_{P}\right)-X_{P}\right)\right)-T$ which does not contain any vertex in $X_{P} \cup\{a, b\}$. This contradicts the assumption that $G-\left(V\left(B_{P}\right)-X_{P}\right)$ is $\left(4, X_{P} \cup\{a, b\}\right)$-connected. Therefore, $G^{\prime}$ is $\left(4, X_{P^{\prime}} \cup\{a, b\}\right)$ connected. 


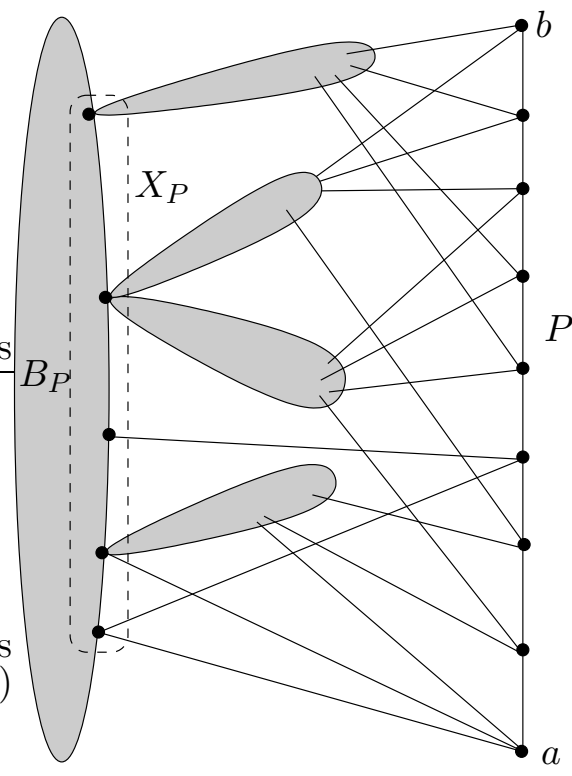

$G$

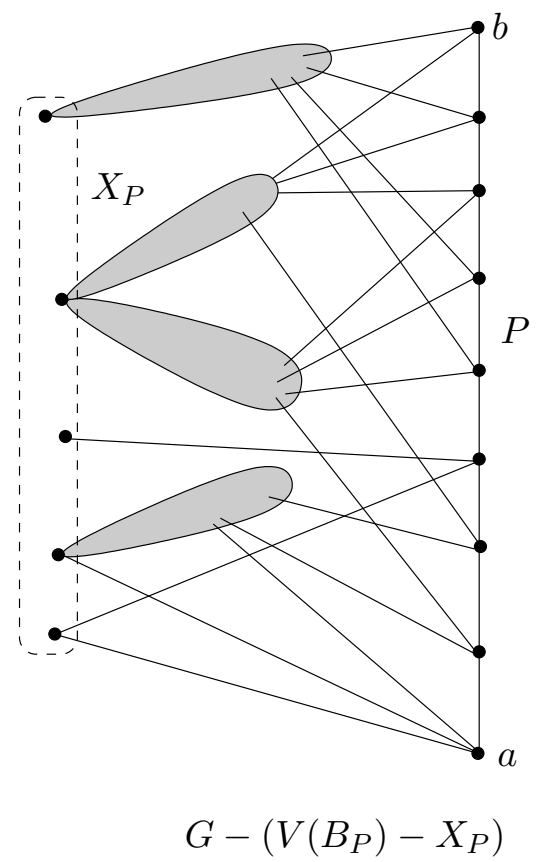

$G-\left(V\left(B_{P}\right)-X_{P}\right)$

Figure 5: $G, a, b, P, B_{P}, R_{P}, X_{P}$ in (3.1).

Let us describe the basic idea of the algorithm which we want to design. At the beginning of each iteration we have a non-separating $a-b$ path $P$ and a nontrivial block $B_{P}$ of $G-V(P)$. The algorithm then tries to find a $B_{P}$-augmenting path $P^{\prime} \in \mathcal{P}_{P}$. If the algorithm finds such a path $P^{\prime}$, then it starts a new iteration with $P^{\prime}$ as $P$ (note that, by Lemma (3.2), $G-\left(V\left(B_{P^{\prime}}\right)-X_{P^{\prime}}\right)$ is $\left(4, X_{P^{\prime}} \cup\{a, b\}\right)$-connected). If the algorithm does not find a $B_{P}$-augmenting path, then it finds a planar $a$ - $b$ chain as required in (1.6).

In order to describe this algorithm more precisely, we need more concepts and notation.

(3.3) Definition. Let $F$ be a subgraph of a graph $K$. An $F$-bridge of $K$ is a subgraph of $K$ which is induced by either (1) an edge in $E(K)-E(F)$ with both ends on $F$, or (2) edges of a component of $K-V(F)$ together with the edges of $K$ from this component to $F$. For an $F$-bridge $B$ of $K$, the set $V(B) \cap V(F)$ is the set of attachments of $B$ on $F$.

(3.4) Notation. Let $\mathcal{B}$ denote the set of $B_{P}$-bridges of $G-V(P)$. For each $B \in \mathcal{B}$, $V\left(B_{P}\right) \cap V(B)$ contains exactly one vertex (which is contained in $X_{P}$ ), and we let $r_{B}$ denote this vertex. For any $x, y \in V(P)$, we denote $x \leq y$ if $x \in V(P[a, y])$. If $x \leq y$ and $x \neq y$, then we write $x<y$. In this case, we say that $x$ is lower than $y$, or $y$ is higher 
than $x$. Since $G$ is $\left(4, X_{P} \cup\{a, b\}\right)$-connected, for each $B \in \mathcal{B}, B-r_{B}$ has at least three neighbors on $P$. Let $l_{B}$ and $h_{B}$ denote the lowest and the highest neighbor of $B-r_{B}$ on $P$, respectively. See Figure 6 for an example.

(3.5) Lemma. The following holds:

(1) $V\left(P\left(l_{B}, h_{B}\right)\right) \neq \emptyset$ and $N_{G}\left(P\left(l_{B}, h_{B}\right)\right) \cap\left(V(B)-\left\{r_{B}\right\}\right) \neq \emptyset$, and

(2) $N_{G}\left(P\left(l_{B}, h_{B}\right)\right) \not \subset V(B) \cup V(P)$.

Proof. It is easy to see that (1) holds because $B-r_{B}$ has at least three neighbors on $P$, and (2) holds because $P$ is an induced path in $G$ and $\left\{r_{B}, l_{B}, h_{B}\right\}$ is not a 3-vertex cut of $G$.

Next, we describe members of $\mathcal{B}$ which we can use to produce a $B_{P}$-augmenting path.

(3.6) Definition. For each vertex $x$ of $G-V(P)$, we define $x^{*}$ as follows. If $x \in V(B)$ for some $B \in \mathcal{B}$, then let $x^{*}:=r_{B}$. If $x \in V\left(B_{P}\right)$, then $x^{*}:=x$. We say that a $B_{P}$-bridge $B \in \mathcal{B}$ is a nice bridge if there exist $x, y \in N_{G}\left(P\left(l_{B}, h_{B}\right)\right)-\left(\left(V(B)-\left\{r_{B}\right\}\right) \cup V(P)\right)$ such that $x^{*} \neq y^{*}$. See Figure 6 for an example.

The next lemma shows that any nice bridge can be used to find a $B_{P}$-augmenting path.

(3.7) Lemma. Let $B \in \mathcal{B}$ be a nice bridge. Then there exists an induced $l_{B}-h_{B}$ path $Q$ in $G\left[V(B) \cup\left\{l_{B}, h_{B}\right\}\right]$ such that $P^{\prime}:=\left(P-V\left(P\left(l_{B}, h_{B}\right)\right)\right) \cup Q$ is a $B_{P}$-augmenting path in $G$. Moreover, such a path $Q$ can be found in $O(|V(G)|+|E(G)|)$ time.

Proof. Since $B$ is a nice bridge, there exist $x, y \in N_{G}\left(P\left(l_{B}, h_{B}\right)\right)-\left(\left(V(B)-\left\{r_{B}\right\}\right) \cup V(P)\right)$ such that $x^{*} \neq y^{*}$. See Figure 6 . Let $G_{B}$ be the subgraph of $G$ induced by $(V(B)-$ $\left.\left\{r_{B}\right\}\right) \cup V\left(P\left[l_{B}, h_{B}\right]\right)$. Since $B$ is a $B_{P}$-bridge, $B-r_{B}$ is connected. Thus, $P\left[l_{B}, h_{B}\right]$ is a non-separating induced path in $G_{B}$. Furthermore, since $G$ is $\left(4, X_{P} \cup\{a, b\}\right)$-connected, for any $T \subset V\left(G_{B}\right)$ with $|T| \leq 2$, every component of $G_{B}-T$ contains a vertex of $V\left(P\left[l_{B}, h_{B}\right]\right)$ (otherwise, $T \cup\left\{r_{B}\right\}$ is a 3 -cut of $G$, and $G-\left(T \cup\left\{r_{B}\right\}\right)$ has a component which contains no vertex in $\left.X_{P} \cup\{a, b\}\right)$. Thus, $G_{B}$ is $\left(3, V\left(P\left[l_{B}, h_{B}\right]\right)\right)$-connected. By Lemma (2.6) (with $G_{B}, l_{B}, h_{B}, P\left[l_{B}, h_{B}\right]$ as $G, a, a^{\prime}, P$ respectively), there exists a non-separating induced $l_{B}-h_{B}$ path $Q$ in $G_{B}$ disjoint from $P\left(l_{B}, h_{B}\right)$. Moreover, such a path $Q$ can be found in $O\left(\left|V\left(G_{B}\right)\right|+\left|E\left(G_{B}\right)\right|\right)$ time. Since $\left|V\left(G_{B}\right)\right|+\left|E\left(G_{B}\right)\right|=$ $O(|V(G)|+|E(G)|)$, such a path $Q$ can be found in $O(|V(G)|+|E(G)|)$ time.

Clearly, the path $P^{\prime}=\left(P-V\left(P\left(l_{B}, h_{B}\right)\right)\right) \cup Q$ is an induced $a-b$ path in $G$.

Let us prove that $P^{\prime}$ is non-separating in $G$. It suffices to prove that for every $v \in$ $V(G)-V\left(P^{\prime}\right)$, there exists a $v-V\left(B_{P}\right)$ path in $G-V\left(P^{\prime}\right)$. This is obvious if $v \in V\left(B_{P}\right)$. So assume $v \notin V\left(B_{P}\right)$. First, suppose $v \in V\left(B^{\prime}\right)$ for some $B^{\prime} \in \mathcal{B}$ with $B^{\prime} \neq B$. Since 


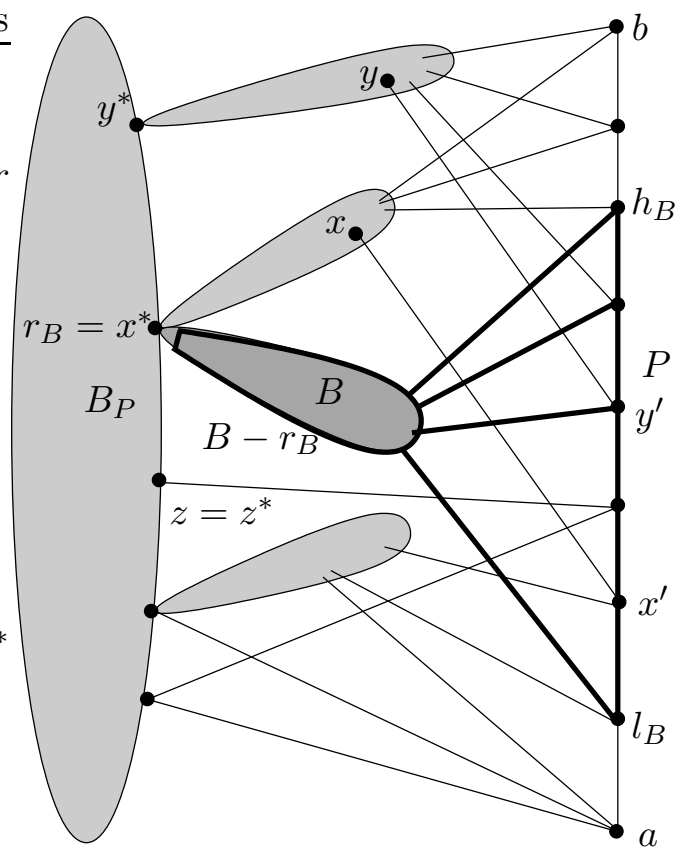

Figure 6: A nice bridge $B$ and the graph $G_{B}$ as defined in the proof of Lemma (3.7) shown in boldface.

$V\left(B^{\prime}\right) \cap V\left(P^{\prime}\right)=\emptyset$, there exists a $v-r_{B^{\prime}}$ path in $B^{\prime}$ (and hence in $G-V\left(P^{\prime}\right)$ ). So we may assume $v \in\left(V(B)-\left\{r_{B}\right\}\right) \cup V\left(P\left(l_{B}, h_{B}\right)\right)$. Since $N_{G}\left(P\left(l_{B}, h_{B}\right)\right) \not \subset V(B) \cup V(P)$ (by (2) of $(3.5))$ and $V\left(Q\left(l_{B}, h_{B}\right)\right) \cap V\left(P\left(l_{B}, h_{B}\right)\right)=\emptyset$, and because $Q$ is a non-separating path in $G_{B}$, there exists a $v-V\left(B_{P}\right)$ path in $G-V\left(P^{\prime}\right)$. Hence, $P^{\prime}$ is non-separating in $G$.

Thus, $P^{\prime} \in \mathcal{P}_{P}$. It remains to show that $\left|V\left(B_{P}\right)\right|<\left|V\left(B_{P^{\prime}}\right)\right|$. Note that $G$ contains disjoint paths $P_{x}$ and $P_{y}$ from $x$ to $x^{*}$ and from $y$ to $y^{*}$, respectively, and $P_{x}$ and $P_{y}$ are disjoint from $P \cup\left(B-r_{B}\right) \cup\left(B_{P}-\left\{x^{*}, y^{*}\right\}\right)$. Let $x^{\prime}, y^{\prime} \in V\left(P\left(l_{B}, h_{B}\right)\right)$ such that $x x^{\prime}, y y^{\prime} \in E(G)$. Then both $B_{P}$ and the path $\left(P_{x} \cup P\left[x^{\prime}, y^{\prime}\right] \cup P_{y}\right)+\left\{x x^{\prime}, y y^{\prime}\right\}$ are contained in $B_{P^{\prime}}$. Hence, $\left|V\left(B_{P}\right)\right|<\left|V\left(B_{P^{\prime}}\right)\right|$, and so, $P^{\prime}$ is a $B_{P^{-a u g m e n t i n g}}$ path.

(3.8) Definition. We say that two $B_{P}$-bridges $B$ and $B^{\prime}$ in $\mathcal{B}$ are overlapping if the paths $P\left[l_{B}, h_{B}\right]$ and $P\left[l_{B^{\prime}}, h_{B^{\prime}}\right]$ have an edge in common. Define an auxiliary graph $\mathcal{K}$ such that $V(\mathcal{K})=\mathcal{B}$, and $B$ and $B^{\prime}$ are adjacent in $\mathcal{K}$ if $B$ and $B^{\prime}$ are overlapping. See Figure 7 for an example.

In what follows we prove several lemmas which will help us find nice bridges (and hence, $B_{P}$-augmenting paths by (3.7)). The following two lemmas appear in [4]. Since their proofs are short, we include them here. 

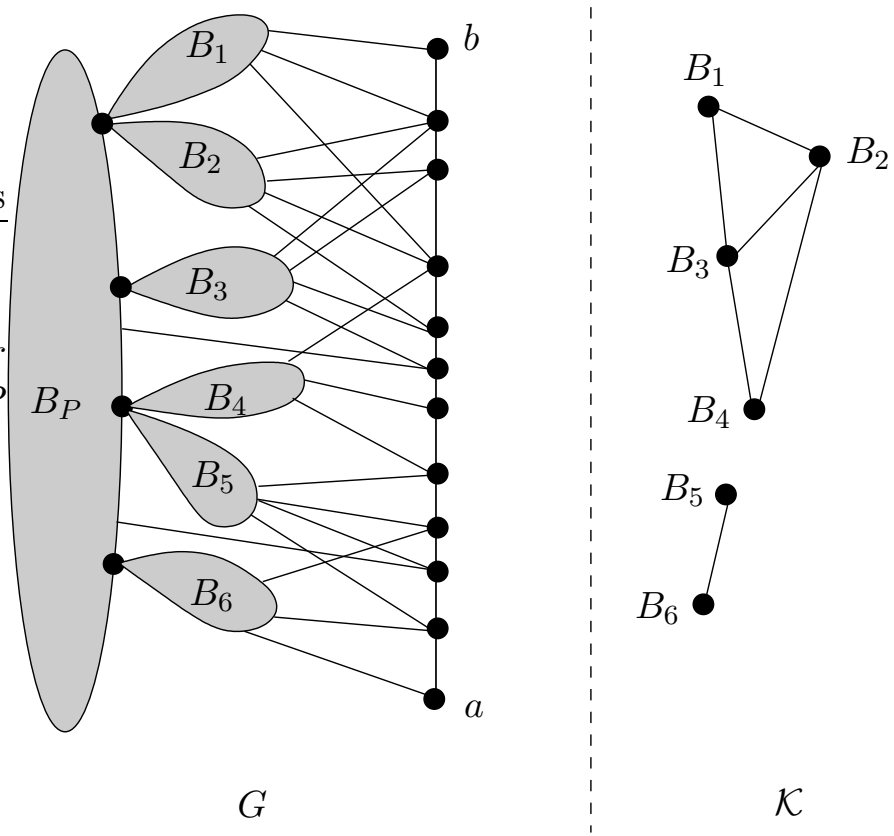

Figure 7: An example of an auxiliary graph $\mathcal{K}$.

(3.9) Lemma. Let $\left(B_{1}, B_{2}, B_{3}\right)$ be an induced path in $\mathcal{K}$ such that $r_{B_{1}} \neq r_{B_{3}}$. Then $B_{2}$ is a nice bridge.

Proof. By the definition of $\mathcal{K}$ and by the assumption that $\left(B_{1}, B_{2}, B_{3}\right)$ is induced in $\mathcal{K}$, $B_{1}$ and $B_{3}$ are not overlapping. So we may assume $l_{B_{1}}<h_{B_{1}} \leq l_{B_{3}}<h_{B_{3}}$. Moreover, $l_{B_{2}}<h_{B_{1}}$ and $l_{B_{3}}<h_{B_{2}}$. Let $x \in V\left(B_{1}\right)-\left\{r_{B_{1}}\right\}$ such that $x h_{B_{1}} \in E(G)$ and let $y \in$ $V\left(B_{3}\right)-\left\{r_{B_{3}}\right\}$ such that $y l_{B_{3}} \in E(G)$. Clearly, $x, y \notin V\left(B_{2}\right)-\left\{r_{B_{2}}\right\}, y \in V\left(P\left(l_{B}, h_{B}\right)\right)$, and $x^{*}=r_{B_{1}} \neq r_{B_{3}}=y^{*}$. Hence, $B_{2}$ is a nice bridge.

(3.10) Lemma. Let $\left(B_{1}, B_{2}, B_{3}\right)$ be a path in $\mathcal{K}$ such that $r_{B_{1}} \neq r_{B_{2}} \neq r_{B_{3}} \neq r_{B_{1}}$. Then one can find in constant time some $i \in\{1,2,3\}$ such that $B_{i}$ is a nice bridge.

Proof. If the path $\left(B_{1}, B_{2}, B_{3}\right)$ is induced in $\mathcal{K}$ then the result follows from the previous lemma. So suppose that $B_{1}, B_{2}, B_{3}$ induces a triangle in $\mathcal{K}$. By symmetry, assume that $P\left[l_{B_{1}}, h_{B_{1}}\right]$ is not properly contained in $P\left[l_{B_{i}}, h_{B_{i}}\right]$ for $i=2,3$ (this can be checked in constant time). Thus, for each $i \in\{2,3\}$, either $l_{B_{i}} \in V\left(P\left(l_{B_{1}}, h_{B_{1}}\right)\right)$, or $h_{B_{i}} \in$ $V\left(P\left(l_{B_{1}}, h_{B_{1}}\right)\right)$, or $P\left[l_{B_{1}}, h_{B_{1}}\right]=P\left[l_{B_{i}}, h_{B_{i}}\right]$. Since $N_{G}\left(P\left(l_{B_{i}}, h_{B_{i}}\right)\right) \cap\left(V\left(B_{i}\right)-\left\{r_{B_{i}}\right\}\right) \neq \emptyset$ (by (1) of (3.5)), it follows that there exist $x \in N_{G}\left(P\left(l_{B_{1}}, h_{B_{1}}\right)\right) \cap\left(V\left(B_{2}\right)-\left\{r_{B_{2}}\right\}\right)$ 
and $y \in N_{G}\left(P\left(l_{B_{1}}, h_{B_{1}}\right)\right) \cap\left(V\left(B_{3}\right)-\left\{r_{B_{3}}\right\}\right)$. Note that $x, y \notin V\left(B_{1}\right)-\left\{r_{B_{1}}\right\}$, and $x^{*}=r_{B_{2}} \neq r_{B_{3}}=y^{*}$. Hence, $B_{1}$ is a nice bridge.

In order to find $B_{P}$-augmenting paths, we need to search the components of $\mathcal{K}$. For convenience, we introduce the following notation.

(3.11) Notation. Let $\mathcal{A}_{1}, \mathcal{A}_{2}, \ldots, \mathcal{A}_{t}$ be the components of the auxiliary graph $\mathcal{K}$. For $j=1, \ldots, t$ let $V_{j}:=\bigcup_{B \in V\left(\mathcal{A}_{j}\right)} V(B)$, let $Q_{j}:=\bigcup_{B \in V\left(\mathcal{A}_{j}\right)} P\left[l_{B}, h_{B}\right]$, and let $R_{\mathcal{A}_{j}}:=$ $\left\{r_{B}: B \in V\left(\mathcal{A}_{j}\right)\right\}$. Note that $V_{j}$ is a subset of $V(G)-\left(V\left(B_{P}\right)-X_{P}\right), Q_{j}$ is a subpath of $P$, and $R\left(\mathcal{A}_{j}\right) \subseteq X_{P}$.

The number of edges in a component of $\mathcal{K}$ can be $O\left(|V(\mathcal{K})|^{2}\right)$, but for our purpose, we need to compute only a spanning tree of each component.

(3.12) Lemma. Algorithm 1 constructs rooted spanning trees $\mathcal{T}_{j}$ of $\mathcal{A}_{j}$ for all $j=$ $1, \ldots, t$, and finds the ends $a_{j}, b_{j}$ of $Q_{j}$ with $a_{j}<b_{j}$, for all $j=1, \ldots, t$. Furthermore, for any $j \in\{1, \ldots, t\}$ and any element $B$ of $V\left(\mathcal{T}_{j}\right)$, the path from the root of $\mathcal{T}_{j}$ to $B$ in $\mathcal{T}_{j}$ is induced in $\mathcal{K}$. Moreover, all $\mathcal{T}_{j}, a_{j}, b_{j}$ can be found in $O(|V(G)|+|E(G)|)$ time.

Proof. The set $\mathcal{Q}$ is implemented as a queue and for each vertex $x$ of the path $P$ we keep a list of $B_{P}$-bridges $B$ such that $l_{B}=x$. The index $k$ is used to avoid re-scanning a vertex more than once. The algorithm is basically a variation of the breadth-first search method and can be implemented to run in $O(|V(G)|)$ time. It is easy to see that each $\mathcal{T}_{j}$ is a spanning tree of a component of $\mathcal{K}$. The first vertex inserted in $\mathcal{T}_{j}$ becomes its root. Furthermore, it is not hard to see that $\mathcal{T}_{j}$ satisfies the following property: for any element $B$ of $V\left(\mathcal{T}_{j}\right)$, the path in $\mathcal{T}_{j}$ from $B$ to the root of $\mathcal{T}_{j}$ is induced in $\mathcal{K}$.

Next, for a component $\mathcal{A}_{j}$ of $\mathcal{K}$ (or more precisely, the spanning tree $\mathcal{T}_{j}$ computed by Algorithm 1) we derive necessary and sufficient conditions for the existence of a nice bridge in $V\left(\mathcal{A}_{j}\right)$, and hence, for the existence of a $B_{P}$-augmenting path by Lemma (3.7). We do this by considering the size of $R_{\mathcal{A}_{j}}$.

(3.13) Lemma. Let $\mathcal{A}_{j}$ be a component of $\mathcal{K}$ such that $\left|R_{\mathcal{A}_{j}}\right| \geq 3$. Then there exists a member of $V\left(\mathcal{A}_{j}\right)$ which is a nice bridge. Moreover, such a member can be found in $O(|V(G)|)$ time.

Proof. We want to show that $\mathcal{T}_{j}$ contains a path $\left(B_{1}, B_{2}, B_{3}\right)$ such that either (i) $\left(B_{1}, B_{2}, B_{3}\right)$ is an induced path in $\mathcal{K}$ and $r_{B_{1}} \neq r_{B_{3}}$, or (ii) $r_{B_{1}} \neq r_{B_{2}} \neq r_{B_{3}} \neq r_{B_{1}}$. For convenience, let $\mathcal{T}:=\mathcal{T}_{j}$. Since $\left|R_{\mathcal{A}_{j}}\right| \geq 3$, there exist members $W, Y$ and $Z$ of $V\left(\mathcal{A}_{j}\right)$ such $r_{W} \neq r_{Y} \neq r_{Z} \neq r_{W}$. Moreover, $W, Y$ and $Z$ can be found in $O(|V(\mathcal{T})|)$ time, and hence, in $O(|V(G)|)$ time. We may assume that $W$ is the root of $\mathcal{T}$. By Lemma (3.12) $\mathcal{T}[W, Y]$ and $\mathcal{T}[W, Z]$ are induced paths in $\mathcal{A}_{j}$. 


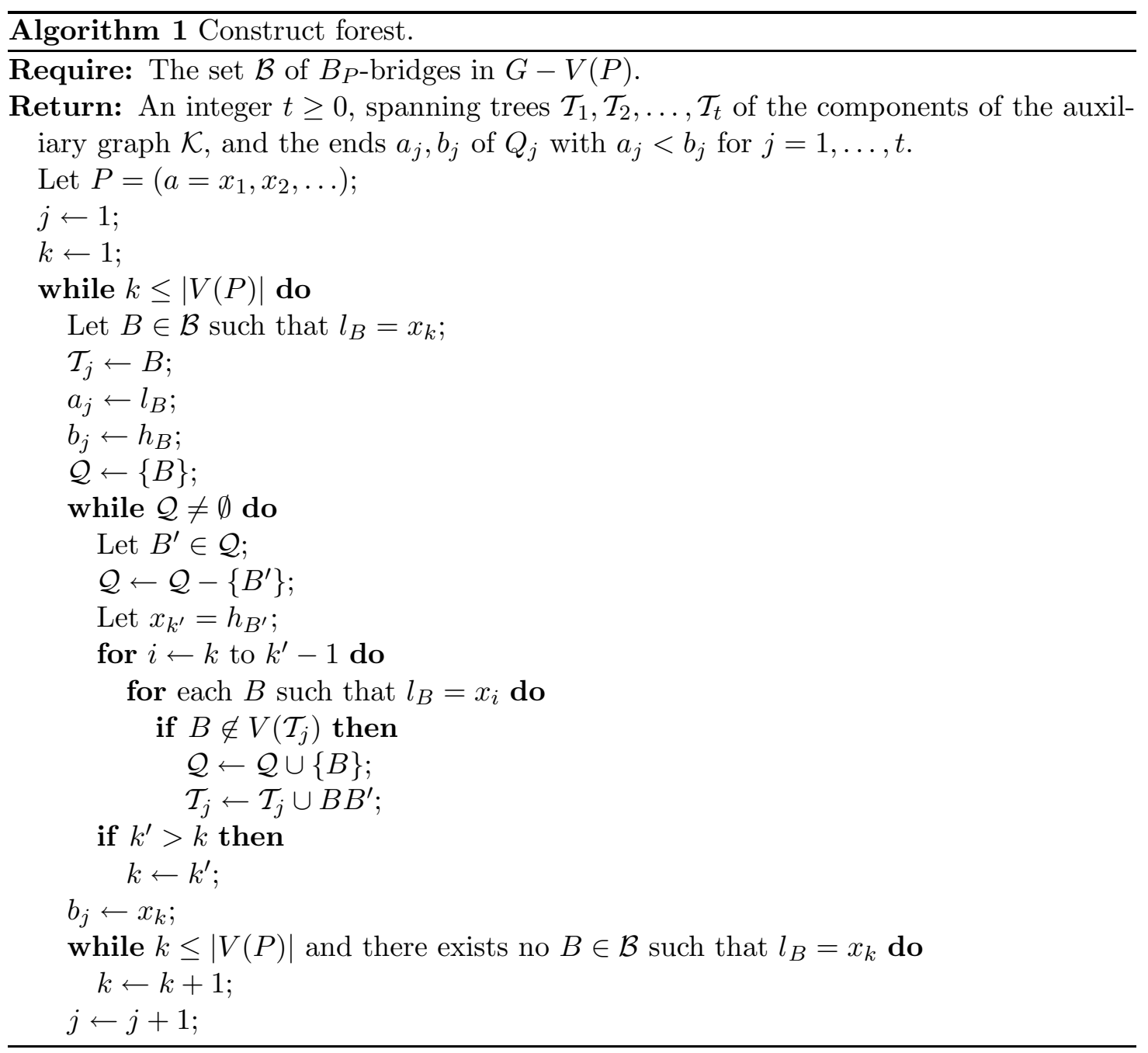

Suppose neither $\mathcal{T}[W, Y]$ nor $T[W, Z]$ contains a path $\left(B_{1}, B_{2}, B_{3}\right)$ satisfying (i) or (ii) above. Because $r_{W} \neq r_{Y}, r_{B} \in\left\{r_{W}, r_{Y}\right\}$ for every member $B$ of $V(\mathcal{T}[W, Y])$ and $r_{B_{1}} \neq$ $r_{B_{2}}$ for every member $B_{1} B_{2}$ of $E(\mathcal{T}[W, Y])$. Similarly, because $r_{W} \neq r_{Z}, r_{B} \in\left\{r_{W}, r_{Z}\right\}$ for any member of $V(\mathcal{T}[W, Z])$ and $r_{B_{1}} \neq r_{B_{2}}$ for any member $B_{1} B_{2}$ of $E(\mathcal{T}[W, Z])$. But since $r_{Z}$ is distinct from $r_{W}$ and $r_{Y}$, it follows that $\mathcal{T}[W, Y] \cup \mathcal{T}[W, Z]$ must contain a path $\left(B_{1}, B_{2}, B_{3}\right)$ which satisfies (i) or (ii). Clearly, this path can be found in $O(|V(G)|)$ time.

By Lemmas (3.9) and (3.10), one of $B_{1}, B_{2}, B_{3}$ is nice bridge, and such a bridge can be found in $O(|V(G)|)$ time. 
If $\left|R_{\mathcal{A}_{j}}\right| \leq 2$ for every $j \in\{1, \ldots, t\}$, then the existence of a nice bridge is not guaranteed. In this case, we will find certain 4-cuts of $G$ which play a fundamental role in the construction of the desired planar $a-b$ chain.

(3.14) Lemma. Let $\mathcal{A}_{j}$ be a component of $\mathcal{K}$ such that $\left|R_{\mathcal{A}_{j}}\right|=1$. Then one of the following holds:

(1) $\left|V\left(\mathcal{A}_{j}\right)\right|=1$ and $\left|\left(N_{G}\left(Q_{j}\left(a_{j}, b_{j}\right)\right) \cap X_{P}\right)-R_{\mathcal{A}_{j}}\right|=1$, or

(2) a member of $V\left(\mathcal{A}_{j}\right)$ is a nice bridge, and it can be found in $O(|V(G)|)$ time.

Proof. We claim that $\left(N_{G}\left(Q_{j}\left(a_{j}, b_{j}\right)\right) \cap X_{P}\right)-R_{\mathcal{A}_{j}} \neq \emptyset$. Otherwise, there exists a component of $G-\left(R_{\mathcal{A}_{j}} \cup\left\{a_{j}, b_{j}\right\}\right)$ not containing any vertex in $X_{P} \cup\{a, b\}$ (because $P$ is induced), which is a contradiction to the assumption that $G$ is $\left(4, X_{P} \cup\{a, b\}\right)$-connected. Thus, let $x \in\left(N_{G}\left(Q_{j}\left(a_{j}, b_{j}\right)\right) \cap X_{P}\right)-R_{\mathcal{A}_{j}}$.

First, suppose $\left|V\left(\mathcal{A}_{j}\right)\right| \geq 2$. Let $B \in V\left(\mathcal{A}_{j}\right)$ such that $x \in N_{G}\left(P\left(l_{B}, h_{B}\right)\right)$. Since $\left|V\left(\mathcal{A}_{j}\right)\right| \geq 2$, there exists $B^{\prime} \in V\left(\mathcal{A}_{j}\right)$ such that $B^{\prime} \neq B$, and $B$ and $B^{\prime}$ overlap. By renaming $B$ and $B^{\prime}$ if necessary, we may assume that $P\left[l_{B}, h_{B}\right]$ is not a proper subpath of $P\left[l_{B^{\prime}}, h_{B^{\prime}}\right]$. Then, either $l_{B^{\prime}} \in V\left(P\left(l_{B}, h_{B}\right)\right)$, or $h_{B^{\prime}} \in V\left(P\left(l_{B}, h_{B}\right)\right)$, or both $l_{B}=l_{B^{\prime}}$ and $h_{B}=h_{B^{\prime}}$. By $(1)$ of $(3.5), N_{G}\left(P\left(l_{B^{\prime}}, h_{B^{\prime}}\right)\right) \cap\left(V\left(B^{\prime}\right)-\left\{r_{B^{\prime}}\right\}\right) \neq \emptyset$. Hence, $P\left(l_{B}, h_{B}\right)$ has a neighbor $y$ such that $y \in V\left(B^{\prime}\right)-\left\{r_{B^{\prime}}\right\}$. Note that $x, y \notin V(B)-\left\{r_{B}\right\}, x^{*}=x \notin V_{j}$ and $y^{*}=r_{B^{\prime}} \in V_{j}$. Thus, $B$ is a nice bridge. Clearly, $B$ can be found in $O(|V(G)|)$ time, and hence, (2) holds.

Now, assume that $\left|V\left(\mathcal{A}_{j}\right)\right|=1$ and $B$ is the only member of $V\left(\mathcal{A}_{j}\right)$. Suppose (1) does not hold. Then $\left|\left(N_{G}\left(Q_{j}\left(a_{j}, b_{j}\right)\right) \cap X_{P}\right)-R_{\mathcal{A}_{j}}\right|>1$. Hence, there exists some $y \in\left(N_{G}\left(Q_{j}\left(a_{j}, b_{j}\right)\right) \cap X_{P}\right)-R_{\mathcal{A}_{j}}$ with $y \neq x$. Then $x, y \notin V(B)-\left\{r_{B}\right\}, x^{*}=x \neq y=y^{*}$, and hence, $B$ is a nice bridge. Again, (2) holds.

(3.15) Lemma. Let $\mathcal{A}_{j}$ be a component of $\mathcal{K}$ such that $\left|R_{\mathcal{A}_{j}}\right|=2$. Then, one of the following holds:

(1) $N_{G}\left(Q_{j}\left(a_{j}, b_{j}\right)\right) \cap X_{P} \subseteq R_{\mathcal{A}_{j}}$, or

(2) a member of $V\left(\mathcal{A}_{j}\right)$ is a nice bridge, and it can be found in in $O(|V(G)|)$ time.

Proof. Suppose that (1) does not hold. Then there exists some $x \in\left(N_{G}\left(Q_{j}\left(a_{j}, b_{j}\right)\right) \cap\right.$ $\left.X_{P}\right)-R_{\mathcal{A}_{j}}$. Note that $x^{*}=x$. Let $B \in V\left(\mathcal{A}_{j}\right)$ such that $x \in N_{G}\left(P\left(l_{B}, h_{B}\right)\right)$. Since $\left|R_{\mathcal{A}_{j}}\right|=2$, we have $\left|V\left(\mathcal{A}_{j}\right)\right| \geq 2$, and hence, there exists $B^{\prime} \in V\left(\mathcal{A}_{j}\right)$ such that $B^{\prime} \neq B$, and $B$ and $B^{\prime}$ overlap. We can rename $B$ and $B^{\prime}$ if necessary so that $P\left[l_{B}, h_{B}\right]$ is not a proper subpath of $P\left[l_{B^{\prime}}, h_{B^{\prime}}\right]$. We can show that $B$ is a nice bridge as in the second paragraph in the proof of Lemma (3.14). 
Before we can fully describe the main algorithm, we need to deal with the situation where (1) of (3.14) or (1) of (3.15) occurs.

(3.16) Definition. Let $\mathcal{A}_{j}$ be a component of $\mathcal{K}$ such that either (i) $\left|R_{\mathcal{A}_{j}}\right|=1$ and $\left|\left(N_{G}\left(Q_{j}\left(a_{j}, b_{j}\right)\right) \cap X_{P}\right)-R_{\mathcal{A}_{j}}\right|=1$, or (ii) $\left|R_{\mathcal{A}_{j}}\right|=2$ and $N_{G}\left(Q_{j}\left(a_{j}, b_{j}\right)\right) \cap X_{P} \subseteq R_{\mathcal{A}_{j}}$. If (i) holds, then let $R_{\mathcal{A}_{j}}:=\left\{c_{j}\right\}$, let $\left(N\left(Q_{j}\left(a_{j}, b_{j}\right)\right) \cap X_{P}\right)-R\left(\mathcal{A}_{j}\right):=\left\{d_{j}\right\}$, and let $G_{j}:=G\left[V_{j} \cup\left\{d_{j}\right\} \cup V\left(P\left[a_{j}, b_{j}\right]\right)\right]-c_{j} d_{j}$. If (ii) holds, then let $R_{\mathcal{A}_{j}}:=\left\{c_{j}, d_{j}\right\}$, and let $G_{j}:=G\left[V_{j} \cup V\left(P\left[a_{j}, b_{j}\right]\right)\right]-c_{j} d_{j}$. In any case, the set $S_{j}:=\left\{a_{j}, b_{j}, c_{j}, d_{j}\right\}$ is a 4-cut in $G$, and $G_{j}-S_{j}$ is a component of $G-S_{j}$. We say that $\mathcal{A}_{j}$ determines the 4-cut $S_{j}$. Since $\mathcal{A}_{j}$ is a component of $\mathcal{K} G_{j}-\left\{c_{j}, d_{j}\right\}$ is 2-connected. See Figure 8.

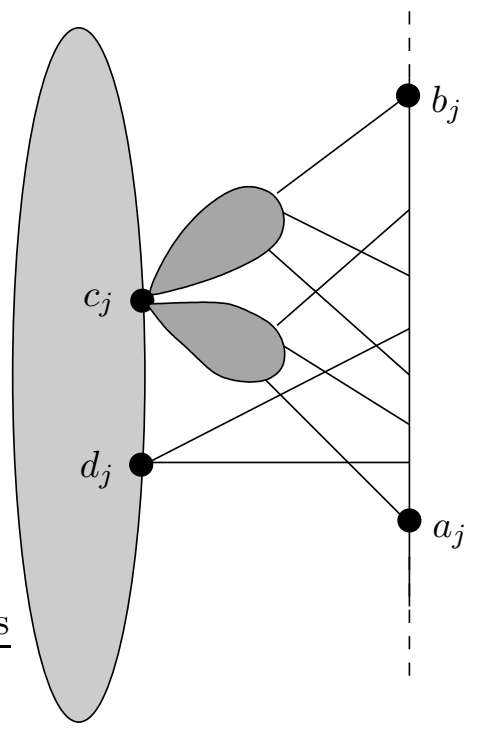

(1)

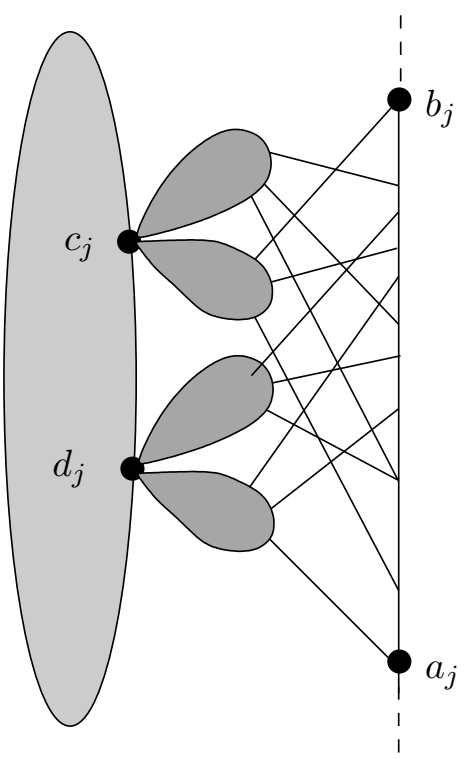

(2)

Figure 8: 4-cuts determined by a component $\mathcal{A}_{j}$ of $\mathcal{K}$.

(3.17) Lemma. Let $\mathcal{A}_{j}$ be a component of $\mathcal{K}$ which determines a 4 -cut $\left\{a_{j}, b_{j}, c_{j}, d_{j}\right\}$. Then one of the following holds:

(1) there exists an induced $a_{j}-b_{j}$ path $Q$ in $G_{j}-\left\{c_{j}, d_{j}\right\}$ such that $\left(P-V\left(P\left(a_{j}, b_{j}\right)\right)\right) \cup Q$ is a $B_{P}$-augmenting path; or

(2) $\left(G_{j}, a_{j}, c_{j}, b_{j}, d_{j}\right)$ is planar. 
Moreover, one can in $O\left(\left|V\left(G_{j}\right)\right|+\left|E\left(G_{j}\right)\right|\right)$ time find a path as in (1) or certify that (2) holds.

Proof. Since $G$ is $\left(4, X_{P} \cup\{a, b\}\right)$-connected, if $T \subset V\left(G_{j}\right)$ with $|T| \leq 3$, then any component of $G_{j}-T$ contains a vertex in $\left\{a_{j}, b_{j}, c_{j}, d_{j}\right\}$. Hence, $G_{j}$ is $\left(4,\left\{a_{j}, b_{j} c_{j}, d_{j}\right\}\right)$ connected. Apply (2.4) with $G_{j}, a_{j}, b_{j}, c_{j}, d_{j}$ as $G, a, a^{\prime}, b, b^{\prime}$ respectively. Then one of the following holds:

(a) there exists a non-separating induced $a_{j}-b_{j}$ path $Q$ in $G_{j}$ such that $V(Q) \cap\left\{c_{j}, d_{j}\right\}=$ $\emptyset$; or

(b) $\left(G_{j}, a_{j}, c_{j}, b_{j}, d_{j}\right)$ is planar.

Moreover, one can in $O\left(\left|V\left(G_{j}\right)\right|+\left|E\left(G_{j}\right)\right|\right)$ time find a path as in (a) or certify that (b) holds.

If (b) occurs, then we have (2). So we may assume that (a) occurs. Let $P^{\prime}:=$ $\left(P-V\left(P\left(a_{j}, b_{j}\right)\right)\right) \cup Q$. Then $P^{\prime}$ is a non-separating induced path in $G$. Moreover, since $\left\{c_{j}, d_{j}\right\}$ is contained in the connected subgraph $G_{j}-V(Q),\left|V\left(B_{P}\right)\right|<\left|V\left(B_{P^{\prime}}\right)\right|$. Thus, $P^{\prime}$ is a $B_{P}$-augmenting path.

We are now ready to prove the main result of this paper: with input $G, a, b, P, B_{P}, X_{P}$, Algorithm 2 returns a planar $a-b$ chain $H$ in $G$ such that $G-V(H)$ is 2-connected and $V\left(B_{P}\right) \subseteq V(G)-V(H)$.

(3.18) Theorem. Algorithm 2 is correct and runs in $O(|V(G)||E(G)|)$ time.

Proof. Let us first prove the correctness of the algorithm.

At the start of each iteration of the main loop, $P$ is a non-separating induced $a$ $b$ path, and $B_{P}$ is a nontrivial block of $G-V(P)$. Moreover, $G-\left(V\left(B_{P}\right)-X_{P}\right)$ is $\left(4, X_{P} \cup\{a, b\}\right)$-connected, where $X_{P}$ is the set consisting of the cut vertices of $G-V(P)$ contained in $V\left(B_{P}\right)$ and the neighbors of $V(P)$ contained in $V\left(B_{P}\right)$. As the algorithm progresses, $\left|V\left(B_{P}\right)\right|$ increases.

If the algorithm stops at line 3, then clearly $G-V(P)$ is 2-connected. Moreover, since $P$ is an induced $a-b$ path, $H:=P$ is also a planar $a-b$ chain whose blocks are all trivial.

If the algorithm stops at line 7 , it returns a subgraph $H$. First, note that $B_{P}=$ $G-V(H)$ is 2-connected. Let us prove that $H$ is a planar $a-b$ chain in $G$. Note that $t \geq 1$. For each $j, 1 \leq j \leq t$, we have that $\left|R\left(\mathcal{A}_{j}\right)\right| \leq 2$ and $\mathcal{A}_{j}$ determines a 4 -vertex cut $S_{j}:=\left\{a_{j}, b_{j}, c_{j}, d_{j}\right\}$ where $c_{j}, d_{j} \in X_{P} \subseteq V\left(B_{P}\right)$. Moreover, $\left(G_{j}, a_{j}, c_{j}, b_{j}, d_{j}\right)$ is planar. Since $\mathcal{A}_{j}$ is a component of $\mathcal{K} G_{j}-\left\{c_{j}, d_{j}\right\}$ is 2-connected. Therefore, $H:=$ $\left(P-\bigcup_{j=1}^{t} V\left(P\left(a_{j}, b_{j}\right)\right)\right) \cup\left(\bigcup_{j=1}^{t} H_{j}\right)$ is a planar $a$ - $b$ chain in $G$. 


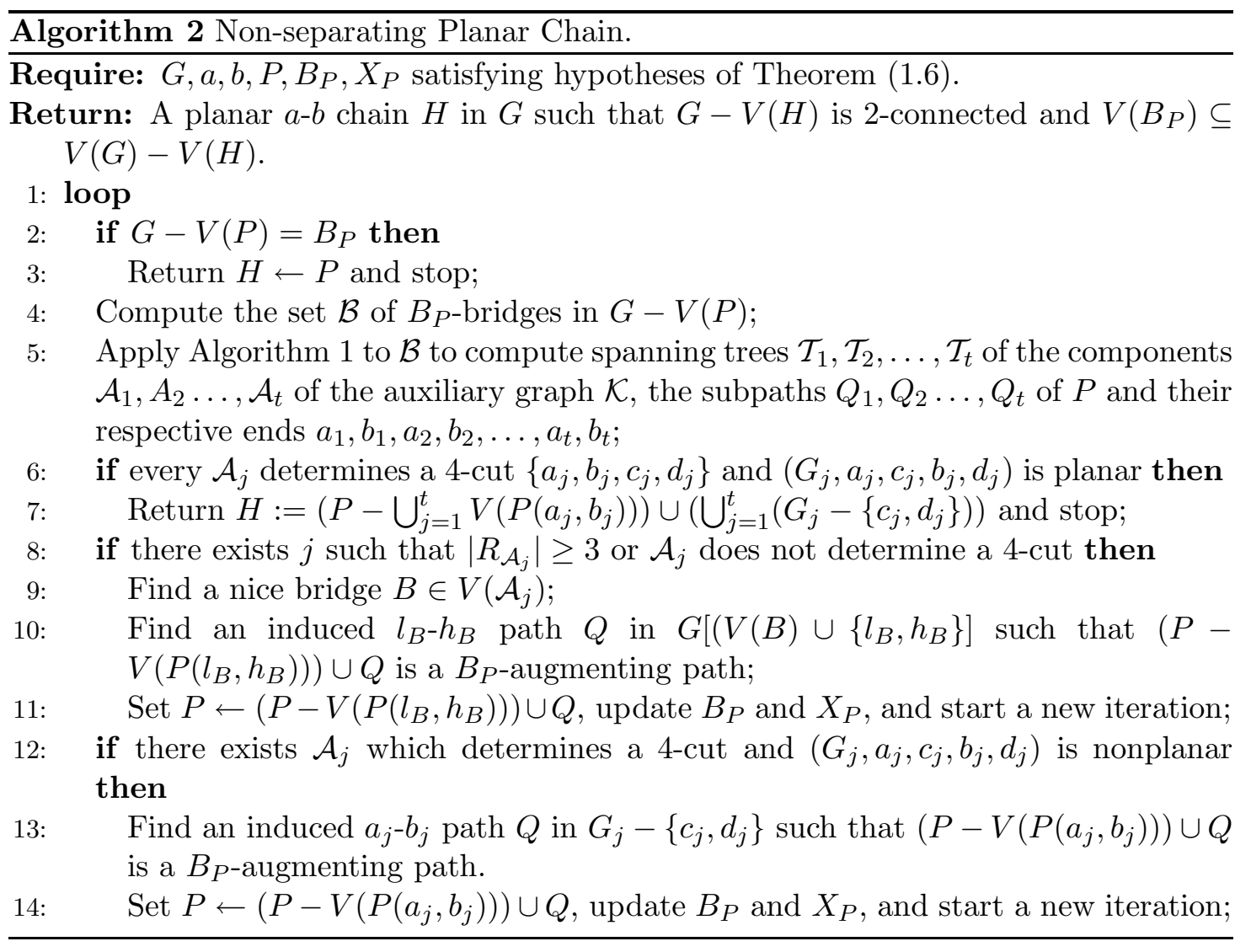

If $B$ is a nice bridge, then by Lemma (3.7) the path $Q$ in line 10 exists and $(P-$ $\left.V\left(P\left(l_{B}, h_{B}\right)\right)\right) \cup Q$ is a $B_{P}$-augmenting path. So, every time the algorithm executes lines 8-11, it increases $\left|V\left(B_{P}\right)\right|$. Moreover, The existence of the nice bridge $B$ on line 9 is guaranteed by (3.13), (2) of (3.14) and (2) of (3.15).

If $\mathcal{A}_{j}$ is a component of $\mathcal{K}$ that determines a 4 -vertex cut $\left\{a_{j}, b_{j}, c_{j}, d_{j}\right\}$ and $\left(G_{j}, a_{j}, c_{j}, b_{j}, d_{j}\right)$ is nonplanar, then by (1) of Lemma (3.17) the path $Q$ in line 13 exists and $\left(P-V\left(P\left(a_{j}, b_{j}\right)\right)\right) \cup Q$ is a $B_{P}$-augmenting path. So when the algorithm executes lines 12-14, it also increases $\left|V\left(B_{P}\right)\right|$.

Finally, Lemma (3.2) guarantees that after the update of $B_{P}$ and $X_{P}$ either in line 11 or in line 14, the hypotheses of Theorem (1.6) still hold in the next iteration. Since $\left|V\left(B_{P}\right)\right|$ increases at each iteration, the loop eventually stops, and hence, Algorithm 2 is correct.

Now, let us verify the complexity of the algorithm.

The loop on line 1 is executed at most $|V(G)|$ times since $\left|V\left(B_{P}\right)\right|$ increases at each iteration. 
The steps in lines 2 and 4 can be performed in $O(|V(G)|+|E(G)|)$ time by standard graph search techniques (for example, see [17]). By Lemma (3.12), the spanning trees $\mathcal{T}_{1}, \ldots, \mathcal{T}_{t}$ (line 5$)$, the paths $Q_{1}, \ldots, Q_{t}$ and their respective ends $a_{1}, b_{1}, \ldots, a_{t}, b_{t}$ can be computed in $O(|V(G)|+|E(G)|)$ time by Algorithm 1 .

The steps in line 6 and line 12 test whether $\left(G_{j}, a_{j}, b_{j}, c_{j}, d_{j}\right)$ is planar. By $(2.4)$ this is equivalent to deciding whether there exists a non-separating induced $a_{j}-b_{j}$ path in $G_{j}$ containing neither $c_{j}$ nor $d_{j}$, and can be done in $O\left(\left|V\left(G_{j}\right)\right|+\left|E\left(G_{j}\right)\right|\right.$ ) (and hence, $O(|V(G)|+|E(G)|))$ time.

Finding a nice bridge $B$ in line 9 can be done in $O(|V(G)|)$ time by (3.13), (2) of (3.14) and (2) of (3.15). The path $Q$ in line 10 can be found in $O(|V(G)|+|E(G)|)$ time by Lemma (3.7). The path $Q$ in line 13 can be found in $O\left(\left|V\left(G_{j}\right)\right|+\left|E\left(G_{j}\right)\right|\right.$ ) (and hence, $O(|V(G)|+|E(G)|)$ time by Lemma (3.17).

Therefore, the running time of Algorithm 2 is $O(|V(G)||E(G)|)$.

\section{Related results}

In this section we present some results related to Theorem (1.6). First, the asymptotic performance of Algorithm 2 can be improved to $O\left(|V(G)|^{2}\right)$ if we add a pre-processing step. Instead of applying the algorithm to $G$, we apply it to a sparse spanning 4-connected subgraph of $G$ with the help from a result of Ibaraki and Nagamochi [8].

(4.1) Theorem. Given a $k$-connected graph $G$, one can find in $O(|V(G)|+|E(G)|)$ time a spanning $k$-connected subgraph of $G$ with at most $k|V(G)|$ edges.

We will use this result and Theorem (1.6) to show how to find in $O\left(|V(G)|^{2}\right)$ time a non-separating "planar cyclic chain" of a 4-connected graph.

The graph obtained from a chain with at least two blocks by identifying its ends is called a "cyclic chain". More precisely, we have the following.

(4.2) Definition. A connected graph $H$ is a cyclic chain if for some integer $k \geq 2$, there exist subgraphs $B_{1}, \ldots, B_{k}$ of $H$ and vertices $v_{1}, \ldots, v_{k}$ of $H$ such that

(i) for $1 \leq i \leq k, B_{i}$ is 2-connected or $B_{i}$ is induced by an edge of $H$,

(ii) $V(H)=\bigcup_{i=1}^{k} V\left(B_{i}\right)$ and $E(H)=\bigcup_{i=1}^{k} E\left(B_{i}\right)$,

(iii) if $k=2$, then $V\left(B_{1}\right) \cap V\left(B_{2}\right)=\left\{v_{1}, v_{2}\right\}$ and $E\left(B_{1}\right) \cap E\left(B_{2}\right)=\emptyset$, and

(iv) if $k \geq 3$, then $V\left(B_{i}\right) \cap V\left(B_{i+1}\right)=\left\{v_{i}\right\}$ for $1 \leq i \leq k$ where $B_{k+1}:=B_{1}$, and $V\left(B_{i}\right) \cap V\left(B_{j}\right)=\emptyset$ for $1 \leq i<i+2 \leq j \leq k$ and $(i, j) \neq(1, k)$. 
We usually fix one of the vertices $v_{1}, \ldots, v_{k}$ as the root of $H$, say $v_{k}$, and we use the notation $H:=v_{0} B_{1} v_{1} \ldots v_{k-1} B_{k} v_{k}$ to indicate that $H$ is a cyclic chain rooted at $v_{0}$ $\left(=v_{k}\right)$. Each subgraph $B_{i}$ is called a piece of $H$. See Figure 9 for an example.

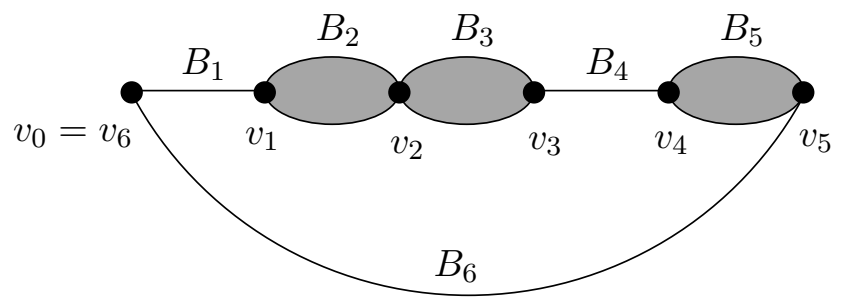

Figure 9: Example of a cyclic chain.

(4.3) Definition. Let $G$ be a graph and let $H:=v_{0} B_{1} v_{1} \ldots v_{k-1} B_{k} v_{k}$ be a cyclic chain rooted at $v_{0}=v_{k}$. If $H$ is an induced subgraph of $G$, then we say that $H$ is a cyclic chain in $G$. We say that $H$ is a planar cyclic chain in $G$, if for each $1 \leq i \leq k$ with $\left|V\left(B_{i}\right)\right| \geq 3$ (or equivalently, $B_{i}$ is 2-connected), there exist distinct vertices $x_{i}, y_{i} \in V(G)-V(H)$ such that $\left(G\left[V\left(B_{i}\right) \cup\left\{x_{i}, y_{i}\right\}\right]-x_{i} y_{i}, x_{i}, v_{i-1}, y_{i}, v_{i}\right)$ is planar, and $B_{i}-\left\{v_{i-1}, v_{i}\right\}$ is a component of $G-\left\{x_{i}, y_{i}, v_{i-1}, v_{i}\right\}$. See Figure 10 for an example.

Our eventual goal is to construct a decomposition of any 4-connected graph into certain chains and find four independent spanning trees. This will be done in forthcoming papers. The next result provides the first chain of such a decomposition. See again Figure 10 for an example.

(4.4) Theorem. Let $G$ be a 4-connected graph and let $r a \in E(G)$. Then there exists a planar cyclic chain $H$ in $G$ rooted at $r$ such that $r a$ is a piece of $H$ and $G-(V(H)-\{r\})$ is 2-connected. Moreover, such a chain can be found in in $O\left(|V(G)|^{2}\right)$ time.

Proof. Let $G$ be a 4-connected graph and let $r a \in E(G)$. By Lemma (4.1), we may assume that $|E(G)|=O(|V(G)|)$. By (1.2), one can find a non-separating induced cycle $C$ in $G$ through $r a$ in $O(|V(G)|+|E(G)|)$ time. Let $P$ denote the path $C-r$ and let $b$ be the end of $P$ other than $a$. Since $C$ is induced, exactly two neighbors of $r$ lie on $P$, namely $a$ and $b$. Thus, $G-V(P)$ is connected and since $C$ is non-separating in $G, r$ is not a cut vertex of $G-V(P)$. Let $B_{P}$ be the block of $G-V(P)$ containing $r$. Note that $N_{G}(r) \subseteq V\left(B_{P}\right) \cup\{a, b\}$. Hence, $B_{P}$ contains more than two vertices because $r$ has degree at least four, and therefore, $B_{P}$ is 2-connected. If $G-V(P)=B_{P}$, then $H:=C$ is a planar cyclic chain rooted at $r$ such that $r a$ is a piece of $H$ and $G-(V(H)-\{r\})$ is 2-connected. So assume that $G-V(P)$ is not 2-connected, that is, $G-V(P) \neq B_{P}$. 


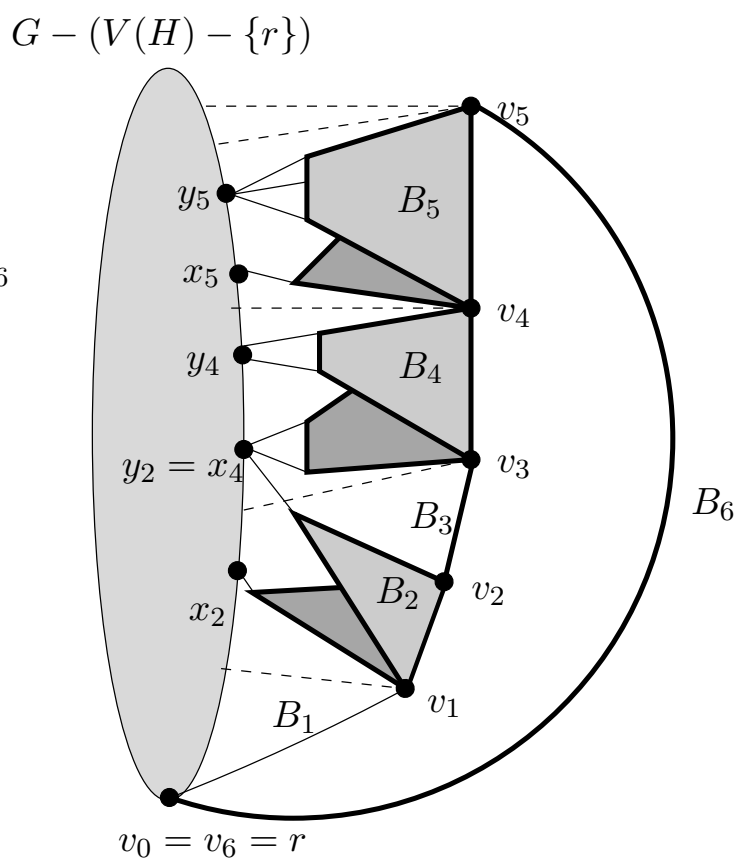

Figure 10: A planar cyclic chain $H:=v_{0} B_{1} v_{1} B_{2} v_{2} B_{3} v_{3} B_{4} v_{4} B_{5} v_{5}$ rooted at $r$ in a graph $G$. The dashed edges may exist or not but they are not part of $H$.

Let $X_{P}$ be the set consisting of the cut vertices of $G-V(P)$ contained in $V\left(B_{P}\right)$ and the neighbors of $V(P)$ contained in $V\left(B_{P}\right)$. Then $G, a, b, P, B_{P}, X_{P}$ satisfy the hypotheses of (1.6). By (1.6) one can find in $O(|V(G)||E(G)|)$ time a planar $a-b$ chain $H^{\prime}$ in $G$ such that $B_{P} \subseteq G-V\left(H^{\prime}\right)$ and $G-V\left(H^{\prime}\right)$ is 2-connected. By our assumption that $|E(G)|=O(|V(G)|)$, such a chain can actually be found in $O\left(|V(G)|^{2}\right)$ time. Since $N_{G}(r) \subseteq V\left(B_{P}\right) \cup\{a, b\}$, we have $r \notin N_{G}\left(H^{\prime}-\{a, b\}\right)$. Therefore, $H:=H^{\prime}+\{r a, r b\}$ is a planar cyclic chain rooted at $r$ such that $r a$ is a piece of $H$ and $G-(V(H)-\{r\})$ is 2-connected.

Remark. The property that $r a$ is a piece in the planar cyclic chain in (4.4) is not necessary for constructing a chain decomposition of a 4-connected graph, but it has an interesting consequence (see Corollary (4.9)). To derive this result from (4.4), we need to introduce some results on Hamilton paths and cycles in planar graphs. Thomassen [19] proved the existence of a special path in a 2-connected planar graph, and later on, Chiba and Nishizeki [3] developed a $O(|V(G)|+|E(G)|)$ algorithm for finding such a path.

(4.5) Theorem. Let $G$ be a 2-connected plane graph with a facial cycle $F$. Let $x \in$ 
$V(F), e \in E(F)$ and $y \in V(G)-\{x\}$. Then $G$ contains an $x$-y path $P$ through $e$ such that

(i) every $P$-bridge of $G$ has at most three attachments on $P$, and

(ii) every $P$-bridge of $G$ containing an edge of $F$ has at most two attachments on $P$.

Moreover, such a path $P$ can be found in $O(|V(G)|+|E(G)|)$ time.

For our purpose, we need the following consequence of (4.5). This was proved by Curran and $\mathrm{Yu}$ [4]. The proof here is essentially the same, but we include it for the sake of completeness.

(4.6) Corollary. Let $(G, a, c, b, d)$ be a planar graph and suppose that $G$ is $(4,\{a, b, c, d\})$-connected. Then there exists a Hamilton $a-b$ path in $G-\{c, d\}$. Moreover, such a path can be found in $O(|V(G)|+|E(G)|)$ time.

Proof. Let $G^{\prime}:=(G-d) \cup\{b c, a c\}$. We first show that $G^{\prime}$ is 2-connected. Suppose on the contrary that $G^{\prime}$ is not 2 -connected. Let $x$ be a cut vertex of $G^{\prime}$. Since $G$ is $(4,\{a, b, c, d\})$ connected, $G-\{c, d\}$ contains an $a-b$ path, and hence, $\{a, b, c\}$ is contained in a cycle in $G^{\prime}$. Therefore, $\{a, b, c\}$ is contained in an $x$-bridge of $G^{\prime}$, and $G^{\prime}$ has another $x$-bridge $B$ such that $(V(B)-\{x\}) \cap\{a, b, c\}=\emptyset$. Hence, $B-x$ is a component of $G-T$, where $T:=\{x, d\}$, and $(V(B)-\{x\}) \cap\{a, b, c\}=\emptyset$, a contradiction.

Note that $G^{\prime}$ is planar, and can be drawn in the plane so that $a c, b c$ and $N_{G}(d)$ are on a facial cycle $F$. Applying (4.5) (with $G^{\prime}, a, c, b c$ as $G, x, y, e$ respectively), $G^{\prime}$ has an $a-c$ path $P$ through $b c$ satisfying (i) and (ii) of (4.5). Moreover, such a path can be found in $O(|V(G)|+|E(G)|)$ time. Note that $a c \notin E(P)$ because $b c \in E(P)$.

We proceed to show that every $P$-bridge of $G^{\prime}$ is induced by a single edge, and so $P$ must be a Hamilton path in $G^{\prime}$. Let $B$ be a $P$-bridge of $G^{\prime}$ such that $V(B)-V(P) \neq \emptyset$, and let $T:=V(B) \cap V(P)$. Since $a, b$ and $c$ are all on $P$, then $\{a, b, c\} \cap V(B) \subseteq T$. Thus, $B-T$ is a component of $G-(T \cup\{d\})$ containing no element of $\{a, b, c, d\}$. If $|T| \leq 2$, then $|T \cup\{d\}| \leq 3$, contradicting our assumption that $G$ is $(4,\{a, b, c, d\})$ connected. Since $P$ satisfies (i) of (4.5), we may assume $|T|=3$. Then by (ii) of (4.5), $E(B) \cap E(F)=\emptyset$, and hence $(V(B)-T) \cap N_{G}(d)=\emptyset$. Therefore, $B-T$ is a component of $G-T$ such that $(V(B)-T) \cap\{a, b, c, d\}=\emptyset$, a contradiction to the assumption that $G$ is $(4,\{a, b, c, d\})$-connected.

Thus, $P-c$ is a Hamilton $a-b$ path in $G-\{c, d\}$. Moreover, by (4.5) such a path can be found in $O(|V(G)|+|E(G)|)$ time.

(4.7) Corollary. Let $G$ be a 4-connected graph and let $H$ be a planar $x$-y chain in $G$. Then there exists a Hamilton $x-y$ path in $H$. Moreover, such a path can be found in $O(|V(H)|+|E(H)|)$ time. 
Proof. Let $H:=v_{0} B_{1} v_{1} \ldots v_{k-1} B_{k} v_{k}$ where $v_{0}=x$ and $v_{k}=y$. Since $H$ is a planar cyclic chain, for each nontrivial block $B_{i}$ of $H$ there exists $u_{i}, w_{i} \in V(G)-V(H)$ such that $\left(G\left[V\left(B_{i}\right) \cup\left\{u_{i}, w_{i}\right\}\right]-u_{i} w_{i}, v_{i-1}, u_{i}, v_{i}, w_{i}\right)$ is planar and $B_{i}-\left\{v_{i-1}, v_{i}\right\}$ is a component of $G-\left\{v_{i-1}, v_{i}, u_{i}, w_{i}\right\}$. Moreover, $G_{i}:=G\left[V\left(B_{i}\right) \cup\left\{u_{i}, w_{i}\right\}\right]-u_{i} w_{i}$ is $\left(4,\left\{v_{i-1}, v_{i}, u_{i}, w_{i}\right\}\right)$ connected. Applying (4.6) to $\left(G_{i}, v_{i-1}, u_{i}, v_{i}, w_{i}\right)$ as $(G, a, c, b, d)$, one can find a Hamilton $v_{i-1}-v_{i}$ path in $B_{i}=G_{i}-\left\{u_{i}, w_{i}\right\}$ in $O\left(\left|V\left(G_{i}\right)\right|+\left|E\left(G_{i}\right)\right|\right)$ time. Therefore, a Hamilton $x-y$ path in $H$ can be found in $O(|V(H)|+|E(H)|)$ time.

By similar argument as in (4.7) we can prove the following.

(4.8) Corollary. Let $G$ be a 4-connected graph and let $H$ be a planar cyclic chain in $G$. Then there exists a Hamilton cycle in $H$. Moreover, such a cycle can be found in $O(|V(H)|+|E(H)|)$ time.

Theorem (4.4), the remark following (4.4) and Corollary (4.8) imply the following interesting result.

(4.9) Corollary. Let $G$ be a 4-connected graph, and $r a \in E(G)$. Then there exists a cycle $C$ in $G$ through ra such that $G-(V(C)-\{r\})$ is 2-connected. Moreover, such a cycle can be found in $O\left(|V(G)|^{2}\right)$ time.

Corollary (4.9) is similar in spirit to (1.2) which was proved by Tutte. Unlike Tutte's result, however, we cannot ask the cycle $C$ in (4.9) to be induced, and, we do not remove the vertex $r$ from the graph. Curran and $\mathrm{Yu}$ ([4], Theorem 1.3) showed that if $G$ is 5-connected and $e \in E(G)$, then $G$ contains an induced cycle $C$ through $e$ such that $G-V(C)$ is 2-connected. All these results are related to the following important open problem. In 1975, Lovász [12] conjectured the following: given any positive integer $k$, there exists some positive integer $f(k)$ with the property that for any given vertices $x$ and $y$ of an $f(k)$-connected graph $G$, there exists an induced $x-y$ path $P$ in $G$ such that $G-V(P)$ is $k$-connected. Thus, Tutte's result solves the case $k=1$, and Curran and Yu's result implies the case $k=2$ which was proved independently by Chen, Gould and $\mathrm{Yu}$ [1] and Kriesell [11]. The conjecture is still open for higher values of $k$.

\section{Appendix}

Proof of Lemma (2.2). First, we prove that exactly one of (1) and (2) holds. Clearly, (1) and (2) are mutually exclusive because of planarity. We know that either (1) or (2) of (2.1) holds. If (1) of (2.1) holds, then (1) of Lemma (2.2) holds. So assume (2) of (2.1) holds. Let $A_{1}, \ldots, A_{k}$ be as described in (2) of (2.1). Then $S \cap A_{i}=\emptyset$ for $1 \leq i \leq k$. Hence, $G\left[A_{i}\right]$ consists of those components of $G-N_{G}\left(A_{i}\right)$ containing no element of $S$, contradicting our assumption that $G$ is $(4, S)$-connected because $\left|N_{G}\left(A_{i}\right)\right| \leq 3$. So no $A_{i}$ can exist. 
Let $G^{\prime}$ be described as in (2) of Theorem (2.1). Observe that $\left(G^{\prime}-\{a b, c d\}, a, c, b, d\right)$ is planar. Since $G^{\prime}-\{a b, c d\}=G$, (2) of Lemma (2.2) holds. Therefore, either (1) or (2) holds.

Let us prove the algorithmic part of Lemma (2.2). First, we give a sketch of Shiloach's algorithm. It has as input a graph $G$ and vertices $a, b, c, d$ of $G$ (with no connectivity hypothesis on $G$ ). The algorithm consists of reductions $\mathrm{R} 1, \ldots, \mathrm{R} 6$ which reduces the general problem to a restricted one.

R1: The algorithm initially reduces the problem to 3-connected graphs, in $O(|V(G)|+$ $|E(G)|)$ time.

$\mathrm{R} 2$ : If the graph is planar, then a specialized $O(|V(G)|+|E(G)|)$ algorithm for 3 -connected planar graphs [14] is used to solve the problem.

R3: Assume that $G$ is nonplanar. This is the most time-consuming step of the algorithm. It reduces the problem using network flow techniques to graphs satisfying some connectivity constraints involving $S:=\{a, b, c, d\}$. Namely, the resulting graph $G$ satisfies the following property: for any subset $S^{\prime}$ of vertices of $G$ with $\left|S^{\prime}\right| \leq 4$, there exist four disjoint paths connecting $S$ to $S^{\prime}$ (these paths can share ends in $S^{\prime}$ though). In fact, this step is not executed at once, but it is interspersed with reductions R4, R5 and R6 in the algorithm. Whenever the algorithm finds a set $S^{\prime}$ which is not connected to $S$ by four disjoint paths, a reduction is performed. The total time spent with these reductions during the whole algorithm is $O(|V(G)||E(G)|)$. For simplicity, suppose that no such set $S^{\prime}$ exists. By Menger's theorem, this is equivalent to saying that $G$ is $(4, S)$-connected. Note that the graph $G$ in the statement of Lemma $(2.2)$ is $(4, S)$-connected.

Thus, so far $G$ is 3-connected, nonplanar, and $(4, S)$-connected. The algorithm then finds a subdivision of a Kuratowski graph $\left(K_{5}\right.$ or $\left.K_{3,3}\right)$. Shiloach gave an $O\left(|V(G)|^{2}\right)$ algorithm to find such a subdivision, but this can be improved as we show below using an algorithm of $\mathrm{Hsu}$ and Shih [6].

R4: If a subdivision of $K_{5}$ is found, Shiloach claims that the required two disjoint paths can be found in $O(|V(G)|+|E(G)|)$ time, using a result of Watkins [21].

R5 and R6: If a subdivision of $K_{3,3}$ is found, then Shiloach's algorithm finds the required two disjoint paths in $O(|V(G)|+|E(G)|)$ time.

Let us show how to improve the running time of the algorithm for $(4, S)$-connected graphs. Let $G$ be a graph, let $S:=\{a, b, c, d\} \subset V(G)$ and suppose that $G$ is $(4, S)$ connected. Let $G^{+}:=G+\{a c, c b, b d, d a\}$. Since $G$ is $(4, S)$-connected, $G^{+}$is $(4, S)$ connected. Because each of $a, b, c, d$ has degree at least three in $G^{+}$, it follows that $G^{+}$ is 3-connected. Moreover, if there exist disjoint paths $P$ and $Q$ in $G^{+}$from $a$ to $b$, and from $c$ to $d$, respectively, then $P$ and $Q$ are both paths in $G$, and vice-versa.

We describe now how to solve the two disjoint paths problem for $G^{+}$in $O(|V(G)|+$ $|E(G)|)$ time. Hsu and Shih $[6]$ developed a $O(|V(H)|+|E(H)|)$ algorithm that given a graph $H$, either finds an embedding of $H$, or finds a subdivision of a Kuratowski graph in $H$. Applying this algorithm to $G^{+}$, either we find an embedding of $G^{+}$, or, a subdivision 
of $K_{5}$ or $K_{3,3}$. If the former occurs, then $G^{+}$is planar, and we can use step R2 to solve the two disjoint paths problem in $O(|V(G)|+|E(G)|)$ time. Otherwise, there exists a subdivision of $K_{5}$ (or $K_{3,3}$ ), and we can use steps R4 (or, R5 and R6, respectively) of Shiloach's algorithm to find the required two disjoint paths. Thus, we can find the two disjoint paths $P$ and $Q$, if they exist, in $O(|V(G)|+|E(G)|)$ time.

\section{References}

[1] G. Chen, R. Gould and X. Yu. Graph connectivity after path removal. Combinatorica, to appear.

[2] J. Cheriyan and S.N. Maheshwari. Finding nonseparating induced cycles and independent spanning trees in 3-connected graphs. J. Algorithms 9 (1988), 507537.

[3] N. Chiba and T. Nishizeki. The hamiltonian cycle problem is linear-time solvable for 4-connected planar graphs. J. Algorithms 10 (1989), 187-211.

[4] S. Curran and X. Yu. Non-separating cycles in 4-connected graphs. Submitted.

[5] D. Dolev, J.Y Halpern, B. Simons and R. Strong. A new look at fault tolerant network routing. Proc. 16th Annual ACM Symp. on Theory of Comput. (1984), $526-535$.

[6] W.-L. Hsu and W.-K. Shih. A new planarity test. Theoretical Comp. Sci. 223 (1999), 179-191.

[7] A. Huck. Independent trees in graphs. Graphs and Combinatorics 10 (1994), $29-45$.

[8] T. Ibaraki and H. Nagamochi. A linear-time algorithm for finding a sparse $k$ connected spanning subgraph of a $k$-connected graph. Algorithmica 7 (1992), $583-596$.

[9] A. Itai and M. Rodeh. The multi-tree approach to reliability in distributed networks. Proc. 25th Annual IEEE Symp. Found. Comp. Sci. (1984), 137-147.

[10] A. Itai and A. Zehavi. Three tree-paths. J. Graph Theory 13 (1989), 175-188.

[11] M. Kriesell. Induced paths in 5-connected graphs. J. Graph Theory 36 (2001), 52-58.

[12] L. Lovász. Problems in Recent Advances in Graph Theory, (ed. M. Fiedler), Academia, Prague, 1975. 
[13] K. Miura, S. Nakano, T. Nishizeki and D. Takahashi. A linear-time algorithm to find four independent spanning trees in four connected planar graphs. Int. J. Found. Comp. Sci. 10 (1999), 195-210.

[14] Y. Perl and Y. Shiloach. Finding two disjoint paths between pairs of vertices in a graph. J. Assoc. Comp. Mach. 25 (1978), 1-9.

[15] P.D. Seymour. Disjoint paths in planar graphs. Discrete Math. 29 (1980), 293309.

[16] Y. Shiloach. A polynomial solution to the undirected two paths problem. $J$. Assoc. Comp. Mach. 27 (1980), 445-456.

[17] R.E. Tarjan. "Data Structures and Network Algorithms", SIAM Monograph, SIAM, Philadelphia, PA, 1983.

[18] C. Thomassen. 2-linked graphs. European J. Combin. 1 (1980), 371-378.

[19] C. Thomassen. A theorem on paths in planar graphs. J. Graph Theory 7 (1983), 169-176.

[20] W.T. Tutte. How to draw a graph. Proc. London Math Soc. 13 (1963), 743-767.

[21] M. Watkins. On the existence of certain disjoint arcs in graphs. Duke Math. J. 35 (1968), 321-346. 